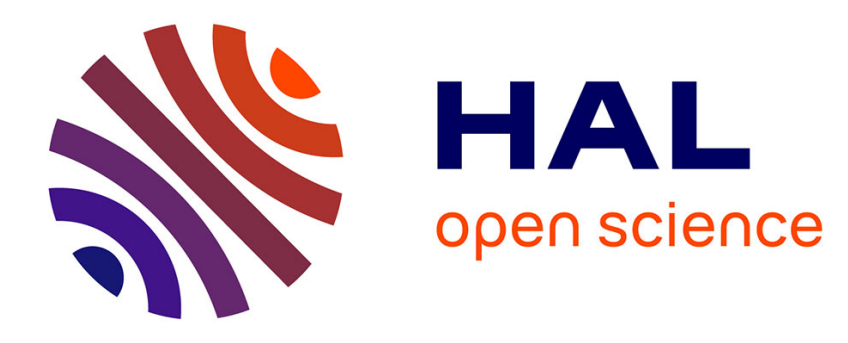

\title{
Optimal dividend and capital structure with debt covenants
}

\author{
Etienne Chevalier, Vathana Ly Vath, Alexandre Roch
}

\section{To cite this version:}

Etienne Chevalier, Vathana Ly Vath, Alexandre Roch. Optimal dividend and capital structure with debt covenants. 2019. hal-02088496

\section{HAL Id: hal-02088496 https://hal.science/hal-02088496}

Preprint submitted on 2 Apr 2019

HAL is a multi-disciplinary open access archive for the deposit and dissemination of scientific research documents, whether they are published or not. The documents may come from teaching and research institutions in France or abroad, or from public or private research centers.
L'archive ouverte pluridisciplinaire HAL, est destinée au dépôt et à la diffusion de documents scientifiques de niveau recherche, publiés ou non, émanant des établissements d'enseignement et de recherche français ou étrangers, des laboratoires publics ou privés. 


\title{
Optimal dividend and capital structure with debt covenants
}

\author{
Etienne Chevalier* Vathana Ly Vath ${ }^{\dagger}$ Alexandre Roch ${ }^{\ddagger}$ \\ April 2, 2019
}

\begin{abstract}
We consider an optimal dividend and capital structure problem for a firm which holds a certain amount of debt to which is associated a financial-ratio covenant between the creditors and the firm. We study optimal policies under a bankruptcy framework using a mixed reduced and structural approach in modelling default and liquidation times. Once in default, the firm is given a grace period during which it may inject more capital to correct the situation. The firm is liquidated if, by the end of the grace period, assets do not exceed the debt.

Under this setup, we maximize the discounted amount of dividends distributed minus the capital injected up to the time of bankruptcy. It gives rise to a two-dimensional singular control problem leading to a non-standard system of variational inequalities. Beyond the usual viscosity characterization, we completely solve this problem and obtain a description of the continuation, dividend and capital injection regions enabling us to fully characterize the optimal policies. We conclude the paper with numerical results and illustrations.
\end{abstract}

Keywords: Viscosity Solutions, System of Variational Inequalities, Singular Controls, Optimal Stopping, Bankruptcy, Debt Covenants.

MSC2000 subject classification: 60G40, 91B70, 93E20.

*Laboratoire de Mathématiques et Modélisation d'Evry, Université Paris Saclay, CNRS et Université d'Evry, France, etienne.chevalier@univ-evry.fr.

${ }^{\dagger}$ Laboratoire de Mathématiques et Modélisation d'Evry, Université Paris Saclay, CNRS and ENSIIE, France, lyvath@ensiie.fr.

‡University of Quebec at Montreal (UQAM) - Faculty of Management, roch.alexandre_@uqam.ca. This author's research was supported by the Natural Sciences and Engineering Research Council of Canada. 


\section{Introduction}

We consider an optimal dividend and capital structure problem for a firm which holds a certain amount of debt to which is associated a financial-ratio covenant between the creditors and the firm. To be more precise, we study dividend and issuance of equity policies under a new bankruptcy framework.

The theory of capital structure is at the center of corporate finance problems. A firm's capital structure is an inherent feature of many mathematical models of credit risk and optimal dividend and investment problems. Made popular by Merton [19], the structural approach to credit risk consists in modelling the dynamics of a firm's assets and debts, and making assumptions about the default and liquidation mechanism. The Merton model assumes a very simple debt design that consists of a single bond with a fixed maturity. The firm defaults if its assets are lower than the face value of the debt at the time of maturity. Black and Cox [2] extended this model by considering the debt structure which allows default at any time before maturity. Under the above and simple definition of a firm's default, the objective of both Merton [19] and Black and Cox [2] is to study the firm's credit risk, in particular the pricing of its debt and its enterprise value.

It is under this simple capital structure framework that most optimal dividend and investment problems have so far been investigated. For instance, Jeanblanc and Shiryaev [11], Asmussen and Taksar [1], Choulli et al. [6], Choulli et al. [7], Sethi and Taksar [21], Décamps and Villeneuve [9], Ly Vath et al. [17], Jin et al. [12], and Chevalier et al. [5] assume that the firm goes bankrupt and ceases operations when its cash reserves (as a proxy for the firm's assets) hits zero. In other words, the level of debt held by a firm is assumed to be zero.

Jeanblanc and Shiryaev [11] and Asmussen and Taksar [1] are among the first studies on optimal dividend policy and consider a stochastic process which represents the cash reserves of the firm. Since then, many studies on dividend policy have been carried solely or in combination with other control problems such as investment policies. Choulli et al. [7] propose a model in which a firm must decide between different production options with differing expected returns, with restrictions on dividend rates. Décamps and Villeneuve [9] and Ly Vath et al. [17] study the interactions between dividend policies and investment decisions in a growth opportunity and under uncertainty whereas Chevalier et al. [5] considers an optimal dividend and investment problem under liquidity constraints.

Chevalier et al. [4] consider a more complex debt structure in which the manager may decide to incur more debt at the cost of higher interest rates. Once again, they assume that the firm defaults and gets liquidated when the cash reserves fall below the current debt level, which is, however, no longer assumed to be zero. Other studies focus on the combined optimal dividend and issuance of equity problem, see for instance Lokka and Zervos [16], Z. Jin et al. [14], Z. Jin and G. Yin [13], and Keppo and Peura [15].

As stated earlier, all the above discussed studies assume that when a firm's assets value reach its debt level, the firm goes bankrupt and gets liquidated. However, in practice, the 
liquidation of a firm is a more complex event. For instance, Makarov et al. [18], and Yildirim [22] price defaultable bonds in a setup in which the liquidation mechanism assumes that the firm's assets must first cross a default threshold and then spend a cumulative amount of time below that barrier in order for the bondholders to be allowed to step in and liquidate the firm. This distinction between default time and liquidation time may be best illustrated by the US bankruptcy code and its Chapters 11 and 7, see for instance Broadie et al. [3]. Indeed, firms may file for protection from liquidation under Chapter 11 and get a grace period to restructure its debts and activities. Chapter 7, i.e. liquidation, is only triggered when the company has not managed to get itself out of default during the allowed time.

In this paper, we are assuming a similar standard bankruptcy process which distinguishes default and liquidation times in the study of our optimal dividend and issuance of equity problem. We consider a firm that holds a certain amount of debt to which is associated a financial-ratio covenant between the creditors and the firm. We assume that the firm may get into default when it is under financial distress, i.e., when its debt-to-assets ratio is less than one. Once in default, the firm is given a grace period during which it can inject more capital to correct the situation. The firm is liquidated if, by the end of the grace period, assets do not exceed the debt. Moreover, as part of the debt covenants, the firm can only pay out dividends to stockholders when its debt-to-assets ratio is less than one. However, in the modelling of default time, we do not consider the usual structural approach as in Broadie et al [3], Makarov et al. [18], and Yildirim [22] which assume that default occurs exactly at the time when an underlying process hits a bound, i.e. the very moment when the firm goes under financial distress. In our study, we consider a reduced model for default which occurs at the first jump of a counting process once an underlying process representing the firm's assets value is below a threshold $D$ corresponding to the firm's debt level. In our case, default time is no longer predictable as required in most credit risk models. Our liquidation time is still based on structural model and occurs when the firm's assets value hits zero or has not reached the upper threshold $D$ before the end of the grace period.

Our objective is to study a dividend and issuance of equity problem under this new framework using a mixed reduced and structural approach in modelling default and liquidation times. To our knowledge, this approach is the first attempt to study optimal dividend and issuance of equity while incorporating such complex capital structure constraints. In terms of mathematical modelling, this gives rise to a two-dimensional singular control problem leading to an atypical and non-standard system of variational inequalities. It is well-known that two-dimensional non-degenerated singular control problems are a major challenge when one expects to get beyond the usual viscosity characterization and obtain a full qualitative description of the optimal policies. This is precisely the results we obtained, a complete description of the continuation, dividend and capital injection regions enabling us to fully describe the optimal policies. This complete qualitative description of different regions may only be obtained by introducing an optimal stopping time on a related reflected diffusion process. Solving this optimal stopping-time problem allows us to characterize the optimal issuance of capital policy during the grace period. 
In the next section, we present the model and some basic properties. In Section 3, we show that the value function is the unique continuous viscosity solution of a system of variational inequalities. In Section 4, we describe the regions of the domain where it is optimal to inject capital and payout dividends. We conclude the paper with numerical illustrations in Section 5.

\section{The model and first properties of the value function}

The firm holds a fixed amount of debt $D \geq 0$. Associated to this debt are covenants between the creditors and the firm. We consider a debt covenant based on the firm's debt to total assets ratio. Under this debt covenant, the firm's financial statement may be audited at any time. If the financial ratio constraint is not satisfied, the firm is declared under default and a grace period starts. Creditors then declare the firm bankruptcy at the end of the grace period if the situation has not improved which means that the ratio constraint has not been satisfied and the default still uncleared before the end of the grace period.

\subsection{The model}

Under the total assets ratio, the firm is in financial difficulty when the total value of its asset at time $t$, denoted by $X_{t}$, is less than $D$, the value of the debt. The firm can increase its capital at any time by issuing more equity, including during a grace period in order to decrease its debt to asset ratio to less than 1 . However, the firm is not allowed to borrow more at this time to increase assets since this action would also increase the debt level. On the other hand, the firm can pay out dividends when it is not in financial distress. The cumulative sum that has been injected into the firm at time $t$ is denoted by $K_{t}$, whereas the total amount of dividends paid up to time $t$ is $Z_{t}$.

When the firm is in financial distress, it may go to default at any time. The probability that a default occurs in the time interval $[t, t+d t)$ is $\lambda d t$, with $\lambda>0$. In other words, the default time is given by the first jump of a Poisson process $N$ with intensity $\lambda$. The firm enters a grace period if $X_{t}<D$ at the default time. When the firm is under default, bankruptcy is defined in terms of a grace period of $\delta$ units of time. In other words, the firm is declared bankrupt when it has spent a continual period of time $\delta$ in financial distress from the last default time or if the value of its assets hits zero (which can happen even if the firm is not currently declared under default). As such, we introduce the default process defined as

$$
d I_{t}=\mathbb{1}_{\left\{X_{t}<D\right\}}\left(1-I_{t}\right) d N_{t}-I_{t} d \mathbb{1}_{\left\{X_{t} \geq D\right\}} .
$$

The default indicator equals 1 when the firm is under default, and 0 otherwise. The process 
$X$ is assumed to satisfy the following stochastic differential equation:

$$
d X_{t}=\mu\left(X_{t}\right) d t+\sigma\left(X_{t}\right) d W_{t}+\left(1-\kappa_{I_{t}}\right) d K_{t}-\left(1+\kappa^{\prime}\right) d Z_{t},
$$

with $\kappa_{0} \leq \kappa_{1}, \kappa^{\prime}$ constants in $(0,1)$. The constants $\kappa_{0}, \kappa_{1}$ and $\kappa^{\prime}$ respectively represent proportional costs that must be paid to inject capital in favorable and unfavorable cases and to payout dividends. The functions $\mu$ and $\sigma$ defined on $\mathbb{R}$ are deterministic, Lipschitz continuous, and satisfy the following linear growth assumption:

$$
|\mu(x)|+|\sigma(x)| \leq C(1+|x|) .
$$

We also define the duration $\Xi_{t}$ that $X$ has spent under $D$ since the last time before $t$ that the firm went to default:

$$
\Xi_{t}=\Xi_{0} \mathbb{1}_{\left\{\inf \left\{s \leq t: I_{s}=0\right\}>\delta-\Xi_{0}\right\}}+t-\sup \left\{s \leq t: I_{s}=0\right\},
$$

with the convention $\sup \emptyset=0$ and $\inf \emptyset=+\infty$. The dynamic of $\Xi$ is the following

$$
d \Xi_{t}=\mathbb{1}_{\left\{I_{t}=1\right\}} d t+\Xi_{t^{-}} d I_{t}
$$

The bankruptcy time is given by

$$
T=\inf \left\{t \geq 0: X_{t}<0 \text { or } \Xi_{t}>\delta\right\} .
$$

We define the state space (with a slight abuse of notation) as $\mathcal{S}=\mathcal{S}^{0} \cup \mathcal{S}^{1}$, with

$$
\mathcal{S}^{0}=[0,+\infty) \quad \text { and } \quad \mathcal{S}^{1}=[0, \delta] \times[0, D] .
$$

The firm is in financial distress and under default $\left(I_{t}=1\right)$ when $\left(\Xi_{t}, X_{t}\right) \in \mathcal{S}^{1} \backslash(\{0\} \times[0, D))$. Note that when $\left(\Xi_{t}, X_{t}\right) \in\{0\} \times[0, D)$ both $I_{t}=1$ and $I_{t}=0$ are possible. Value functions of the optimization problem are then respectively defined by

$$
\begin{aligned}
v_{0}(x) & :=\sup _{(Z, K) \in \mathcal{A}} \mathbb{E}_{x, 0} \int_{0}^{T} e^{-\rho s} d\left(Z_{s}-K_{s}\right), \quad x \in \mathcal{S}^{0}, \\
v_{1}(\varsigma, x) & :=\sup _{(Z, K) \in \mathcal{A}} \mathbb{E}_{\varsigma, x, 1} \int_{0}^{T} e^{-\rho s} d\left(Z_{s}-K_{s}\right), \quad(\varsigma, x) \in \mathcal{S}^{1},
\end{aligned}
$$

when the firm is not under default $(I=0)$ and when it is under default $(I=1)$.

Here, $\rho>0$ is a discount rate, $\mathbb{E}_{\varsigma, x, i}$ is the conditional expectation given that $X_{0-}=x$, $\Xi_{0}=\varsigma$ and $I_{0-}=i$ ( $\varsigma$ is omitted when the firm is not under default), and

$$
\mathcal{A}=\left\{(Z, K) \in \mathcal{I}_{\mathbb{F}}^{2}: \forall t \geq 0, Z_{t}-Z_{t^{-}} \leq\left(X_{t^{-}}-D\right)^{+} \text {and } \int_{0}^{+\infty} \mathbb{1}_{\left\{I_{t}=1\right\}} d Z_{t}=0\right\}
$$


is the set of admissible dividend and capital injection policies. $\mathcal{I}_{\mathbb{F}}$ denotes the set of non decreasing and $\mathbb{F}$-predictable processes. We also use the notation

$$
\mathcal{A}^{0}=\{K:(Z, K) \in \mathcal{A}, Z \equiv 0\} .
$$

\subsection{Upper bound for the value function}

Throughout the paper, we make the following standing assumption:

$$
\sup _{x \geq 0}(\mu(x)-\rho x)<+\infty .
$$

This condition will guarantee that the value of the firm is finite as in the next proposition.

Proposition 2.1. For any $x \geq 0$, we set $f(x):=\frac{x}{1+\kappa^{\prime}}+\frac{1}{\rho} \sup _{x \geq 0}(\mu(x)-\rho x)$, and we have

$$
v_{0}(x) \leq f(x) \text { on } \mathcal{S}^{0} \quad \text { and } \quad v_{1}(\varsigma, x) \leq f(x) \text { on } \mathcal{S}^{1} .
$$

Proof: We introduce an arbitrary control $\alpha=(Z, K) \in \mathcal{A}$. For $m>0$, we set

$$
\theta_{m}=\inf \left\{t \geq 0: \Xi_{t} \geq \delta-\frac{1}{m}, \text { or } X_{t} \leq 1 / m\right\} .
$$

Notice that $\theta_{m} \nearrow T$ a.s. when $m$ goes to infinity. We apply Itô's formula to $\left(e^{-\rho t} f\left(X_{t}\right)\right)_{t \geq 0}$ between 0 and $\theta_{m}$ :

$$
\begin{aligned}
e^{-\rho \theta_{m}} f\left(X_{\theta_{m}}\right)= & f\left(X_{0}\right)+\int_{0}^{\theta_{m}} e^{-\rho t}(\mathcal{L} f-\rho f)\left(X_{t}\right) d t-\int_{0}^{\theta_{m}} e^{-\rho t} f^{\prime}\left(X_{t}\right) d\left(Z_{t}^{c}-K_{t}^{c}\right) \\
& +\sum_{0 \leq t \leq \theta_{m}} e^{-\rho t}\left[f\left(X_{t}\right)-f\left(X_{t^{-}}\right)\right]+\int_{0}^{\theta_{m}} e^{-\rho t} \sigma\left(X_{t}\right) f^{\prime}\left(X_{t}\right) d B_{t} .
\end{aligned}
$$

where $\mathcal{L} f(x):=\frac{\sigma(x)^{2}}{2} \frac{\partial^{2} f}{\partial x^{2}}+\mu(x) \frac{\partial f}{\partial x}, Z^{c}$ and $K^{c}$ are the continuous parts of $Z$ and $K$. As $f(x):=\frac{x}{1+\kappa^{\prime}}+C$ where $C:=\frac{1}{\rho} \sup _{x \geq 0}(\mu(x)-\rho x)$, we have

$$
\begin{aligned}
e^{-\rho \theta_{m}} f\left(X_{\theta_{m}}\right)= & f\left(X_{0}\right)+\frac{1}{1+\kappa^{\prime}} \int_{0}^{\theta_{m}} e^{-\rho t}\left(\mu\left(X_{t}\right)-\rho X_{t}-\rho C\right) d t \\
& -\frac{1}{1+\kappa^{\prime}} \int_{0}^{\theta_{m}} e^{-\rho t} d\left(\left(1+\kappa^{\prime}\right) Z_{t}^{c}-\left(1-\kappa_{I_{0}}\right) K_{t}^{c}\right) \\
& +\frac{1}{1+\kappa^{\prime}} \sum_{0 \leq t \leq \theta_{m}} e^{-\rho t}\left[X_{t}-X_{t^{-}}\right] \\
& +\frac{1}{1+\kappa^{\prime}} \int_{0}^{\theta_{m}} e^{-\rho t} \sigma\left(X_{t}\right) d B_{t} .
\end{aligned}
$$


As $\kappa^{\prime}, \kappa_{0}$, and $\kappa_{1}>0$, by taking expectation in the above equation, we get

$$
\mathbb{E}\left[e^{-\rho \theta_{m}} f\left(X_{\theta_{m}}\right)\right] \leq f\left(X_{0}\right)-\mathbb{E}\left[\int_{0}^{\theta_{m}} e^{-\rho t} d\left(Z_{t}-K_{t}\right)\right]
$$

Recall that $f(y) \geq 0$, by sending $m$ to infinity, it follows from Fatou's lemma that:

$$
f\left(X_{0}\right) \geq \mathbb{E}\left[\int_{0}^{T} e^{-\rho t} d\left(Z_{t}-K_{t}\right)+e^{-\rho T} f\left(X_{T}\right)\right] \geq \mathbb{E}\left[\int_{0}^{T} e^{-\rho t} d\left(Z_{t}-K_{t}\right)\right]
$$

As this is true for any initial state $\left(I_{0}, \Xi_{0}, X_{0}\right)$ and any arbitrary control $(Z, K) \in \mathcal{A}$. This concludes the proof.

\subsection{Dynamic programming}

We start this section with the introduction of a notation that will be used throughout the paper.

Hitting times: For $y \in[0, D]$, we define the hitting times

$$
\theta^{y,-}=\inf \left\{t \geq 0: X_{t} \leq y\right\} \quad \text { and } \quad \theta^{y,+}=\inf \left\{t \geq 0: X_{t} \geq y\right\}
$$

In this setting, the dynamic programming principle (DPP) that we shall use is the following:

\section{Dynamic Programming Principle (DPP):}

Let $\tau=\inf \left\{t \geq 0: I_{t}=1\right\}$ be the first default time. For all stopping times $\nu$ and all $x \in \mathcal{S}^{0}$, we have the following dynamic programming principle for $v_{0}$ :

$$
\begin{aligned}
v_{0}(x)=\sup _{(Z, K) \in \mathcal{A}} \mathbb{E}_{x, 0}\left[\int_{0}^{\nu \wedge \tau \wedge T} e^{-\rho s} d\left(Z_{s}-K_{s}\right)\right. & +e^{-\rho \nu} v_{0}\left(X_{\nu}\right) \mathbb{1}_{\{\nu<\tau \wedge T\}} \\
& \left.+e^{-\rho \tau} v_{1}\left(0, X_{\tau}\right) \mathbb{1}_{\{\tau \leq \nu \wedge T\}}\right] .
\end{aligned}
$$

For all $(\varsigma, x) \in \mathcal{S}^{1}$ and stopping times $\nu$ taking values in $[0, \delta-\varsigma]$, we have the following dynamic programming principle for $v_{1}$ :

$$
\begin{aligned}
v_{1}(\varsigma, x)=\sup _{(Z, K) \in \mathcal{A}} \mathbb{E}_{\varsigma, x, 1}\left[-\int_{0}^{\nu \wedge \theta^{D,+} \wedge T} e^{-\rho t} d K_{t}\right. & +e^{-\rho \nu} v_{1}\left(\Xi_{\nu}, X_{\nu}\right) \mathbb{1}_{\left\{\nu<T \wedge \theta^{D,+}\right\}} \\
& \left.+e^{-\rho \theta^{D,+}} v_{0}\left(X_{\theta^{D,+}}\right) \mathbb{1}_{\left\{\theta^{D,+} \leq T \wedge \nu\right\}}\right] .
\end{aligned}
$$

Remark 2.1. Note that $\left\{\theta^{D,+}=T\right\}=\emptyset$ and $v_{1}(\delta, x)=g(x)$, where we have set

$$
g(x):=\max \left(v_{0}(D)-(D-x) /\left(1-\kappa_{1}\right), 0\right), \quad \text { on }[0, D]
$$


Indeed, for $x \in[0, D]$, one could issue capital up to $D$ or go to bankruptcy, so we obviously have $v_{1}(\delta, x) \geq g(x)$. Moreover, equation (2.13) gives that

$$
\begin{aligned}
v_{1}(\delta, x) & =\max \left(\sup _{a \geq 0}\left[-a+v_{0}\left(D+\left(1-\kappa_{1}\right) a\right)\right]-\frac{D-x}{1-\kappa_{1}} ; 0\right) \\
& \leq \max \left(\sup _{a \geq 0}\left[-a+v_{0}(D)+\frac{1-\kappa_{1}}{1-\kappa_{0}} a\right]-\frac{D-x}{1-\kappa_{1}} ; 0\right) \\
& =\max \left(v_{0}(D)-\frac{D-x}{1-\kappa_{1}} ; 0\right)=g(x) .
\end{aligned}
$$

Under default, we guess that, when it is optimal to clear the default, capital has to be issued up to $D$ and not more. Indeed when default is cleared the cost of issuing new capital is then lower. Consequently, we now prove that we can restrict the set of admissible strategies to these strategies. The following Corollary of the DPP states that if default is cleared at time $t$ before $\Xi_{t}=\delta$, we can assume that $X_{t}=D$.

Corollary 2.1. For all $(\varsigma, x) \in \mathcal{S}^{1}$ and stopping times $\nu$,

$$
\begin{aligned}
v_{1}(\varsigma, x)=\tilde{v}_{1}(\varsigma, x):= & \sup _{K \in \mathcal{A}^{0}} \mathbb{E}_{\varsigma, x, 1}\left[-\int_{0}^{\left(\nu \wedge \theta^{D,+} \wedge T\right)^{-}} e^{-\rho t} d K_{t}-e^{-\rho \nu} \Delta K_{\nu} \mathbb{1}_{\left\{\nu<T \wedge \theta^{D,+} \wedge(\delta-\varsigma)\right\}}\right. \\
& \left.+e^{-\rho \nu} v_{1}\left(\Xi_{\nu}, X_{\nu}\right) \mathbb{1}_{\left\{\nu<T \wedge \theta^{D,+} \wedge(\delta-\varsigma)\right\}}+e^{-\rho \nu} g\left(X_{\nu^{-}}\right) \mathbb{1}_{\left\{\nu=(\delta-\varsigma) \wedge \theta^{D,+}<T\right\}}\right] .
\end{aligned}
$$

Note that, starting from $(\varsigma, x) \in \mathcal{S}^{1}$, we have $\Xi_{\nu}=\nu+\varsigma$ on $\left\{\nu<T \wedge \theta^{D,+} \wedge(\delta-\varsigma)\right\}$.

Proof: On $[0, D]$, we have $v_{0}(x) \leq v_{0}(D)+(x-D) /\left(1-\kappa_{0}\right) \leq v_{0}(D)+(x-D) /\left(1-\kappa_{1}\right)$. Hence, by Equation 2.13, on $\mathcal{S}^{1}, v_{1}$ is less or equal to $\tilde{v}_{1}$.

For any $K \in \mathcal{A}^{0}$, consider the strategy $\tilde{K}$ such that $\tilde{K}_{s}=K_{s}$ for all $s<\delta-\varsigma$, and

$$
\tilde{K}_{s}= \begin{cases}K_{\delta-\varsigma}-\left(X_{(\delta-\varsigma)}-D\right)^{+} /\left(1-\kappa_{1}\right), & \text { if } s=\delta-\varsigma \\ K_{\theta^{D,+}}-\left(X_{\theta^{D,+}}-D\right) /\left(1-\kappa_{1}\right), & \text { if } s=\theta^{D,+} \\ K_{s}, & \text { otherwise. }\end{cases}
$$

Then, $(Z, \tilde{K}) \in \mathcal{A}$ and, if we denote by $\tilde{X}$ the process $X$ controlled by $\tilde{K}, \tilde{T}$ its bankruptcy time and $\tilde{\theta}^{D,+}$ its associated hitting time of $D$. Notice that we have $\tilde{\theta}^{D,+}=\theta^{D,+}$ and $\tilde{T}=T$. Therefore, we have 


$$
\begin{aligned}
v_{1}(\varsigma, x) \geq & \mathbb{E}\left[-\int_{0}^{\nu \wedge \tilde{\theta}^{D,+} \wedge \tilde{T}} e^{-\rho t} d \tilde{K}_{t}+e^{-\rho \nu} v_{1}\left(\Xi_{\nu}, \tilde{X}_{\nu}\right) \mathbb{1}_{\left\{\nu<\tilde{T} \wedge \tilde{\theta}^{D,+}\right\}}+e^{-\rho \tilde{\theta}^{D,+}} v_{0}\left(\tilde{X}_{\tilde{\theta}^{D,+}}\right) \mathbb{1}_{\left\{\tilde{\theta}^{D,+}<\tilde{T} \wedge \nu\right\}}\right] \\
= & \mathbb{E}\left[-\int_{0}^{\left(\nu \wedge \theta^{D,+} \wedge T\right)^{-}} e^{-\rho t} d K_{t}-e^{-\rho \nu}\left(\Delta K_{\nu}-v_{1}\left(\Xi_{\nu}, X_{\nu}\right)\right) \mathbb{1}_{\left\{\nu<T \wedge \theta^{D,+} \wedge(\delta-\varsigma)\right\}}\right. \\
& \left.+e^{-\rho\left(\theta^{D,+} \wedge(\delta-\varsigma)\right)} g\left(X_{\theta^{D,+} \wedge(\delta-\varsigma)}\right) \mathbb{1}_{\left\{\theta^{D,+} \wedge(\delta-\varsigma)<T \wedge \nu\right\}}\right] .
\end{aligned}
$$

Therefore, $v_{1}(\varsigma, x) \geq \tilde{v}_{1}(\varsigma, x)$.

\subsection{Analytical properties of the value functions}

In this section, we study the limit of the value function on the domain boundaries, establish some monotonicity results and prove that the value functions are continuous.

Proposition 2.2. The function $v_{1}$ satisfies the following limits on the boundary of $\mathcal{S}^{1}$ :

i) $\lim _{h \downarrow 0} \sup _{x \in[0, D]}\left|v_{1}(\delta-h, x)-v_{1}(\delta, x)\right|=0$, and $v_{1}(\delta, x)=g(x)$, for all $0 \leq x \leq D$,

ii) $\lim _{h \downarrow 0} v_{1}(\varsigma, D-h)=v_{0}(D)=v_{1}(\varsigma, D)$ for all $0 \leq \varsigma \leq \delta$,

iii) $\lim _{h \downarrow 0} v_{1}(\varsigma, h)$ exists for all $0 \leq \varsigma \leq \delta$.

Proof: We first note that $g(x) \leq v_{1}(\varsigma, x)$ on $\mathcal{S}^{1}$. Indeed, if $x>D-v_{0}(D)\left(1-\kappa_{1}\right)$, we consider a strategy $K$ such that $\Delta K_{0}=(D-x) /\left(1-\kappa_{1}\right)$ and obtain

$$
v_{1}(\varsigma, x) \geq-\Delta K_{0}+v_{0}\left(x+\left(1-\kappa_{1}\right) \Delta K_{0}\right)=-(D-x) /\left(1-\kappa_{1}\right)+v_{0}(D)=g(x) .
$$

Otherwise, if $x<D-v_{0}(D)\left(1-\kappa_{1}\right), g(x)=0$, and obviously $v_{1}(\varsigma, x) \geq g(x)$.

Let $0<h<\delta$ and $0<\varepsilon<x$. Define $\nu=h \wedge \theta^{D,+} \wedge \theta^{\varepsilon,-}$. From Corollary 2.1, it follows that

$$
\begin{aligned}
v_{1}(\delta-h, x)=\sup _{(Z, K) \in \mathcal{A}} \mathbb{E}[ & -\int_{0}^{\nu^{-}} e^{-\rho t} d K_{t}-e^{-\rho \nu}\left(\Delta K_{\nu}-v_{1}\left(\Xi_{\nu}, X_{\nu}\right)\right) \mathbb{1}_{\left\{\nu=\theta^{\epsilon,-}<h \wedge \theta^{D,+}\right\}} \\
& \left.+e^{-\rho \theta^{D,+}} g(D) \mathbb{1}_{\left\{\theta^{D,+} \leq h \wedge \theta^{\epsilon,-}\right\}}+e^{-\rho h} g\left(X_{h}\right) \mathbb{1}_{\left\{h<\theta^{D,+}\right\}}\right] .
\end{aligned}
$$

Hence, if we define the process $\tilde{X}:=\left(D \wedge X_{t}\right)_{t \geq 0}$ and set

$$
\tilde{v}_{h}(x)=\sup _{K \in \mathcal{A}^{0}} \mathbb{E}_{\delta-h, x}\left[e^{-\rho \nu}\left(-K_{\nu}+g\left(\tilde{X}_{\nu}\right)+v_{1}\left(\Xi_{\theta^{\varepsilon,-}}, \varepsilon\right) \mathbb{1}_{\left\{\nu=\theta^{\epsilon,-}<h \wedge \theta^{D,+}\right\}}\right)\right],
$$

we obviously have that $v_{1}(\delta-h, x) \leq \tilde{v}_{h}(x)$. Also, it is clear that $g(x) \leq \tilde{v}_{h}(x)$.

It only remains to show that $\tilde{v}_{h}(x)$ converges to $g(x)$ uniformly in $x$, as $h \rightarrow 0$. In order to do so, first note that

$$
g\left(\tilde{X}_{\nu}\right)-K_{\nu} \leq g\left(x+\int_{0}^{\nu}\left(\mu\left(X_{s}\right) d s+\sigma\left(X_{s}\right) d W_{s}\right)\right) .
$$


Consequently, it follows from Proposition (2.1) that there exists $C>0$ such that

$$
\begin{aligned}
\tilde{v}_{h}(x) & \leq \sup _{K \in \mathcal{A}^{0}} \mathbb{E}\left[g\left(x+\int_{0}^{\nu}\left(\mu\left(X_{s}\right) d s+\sigma\left(X_{s}\right) d W_{s}\right)\right)+C \mathbb{1}_{\left\{\nu=\theta^{\epsilon,-}<h\right\}}\right] \\
& \left.\leq g(x)+\frac{1}{1-\kappa_{1}} \sup _{K \in \mathcal{A}^{0}} \mathbb{E} \mid \int_{0}^{\nu}\left(\mu\left(X_{s}\right) d s+\sigma\left(X_{s}\right) d W_{s}\right)\right)+C \mathbb{1}_{\left\{\nu=\theta^{\epsilon,-}<h\right\}} \mid .
\end{aligned}
$$

It follows from the linear growth assumption (2.3) that $\sup _{s}\left|\mu\left(X_{s}\right)\right|+\left|\sigma\left(X_{s}\right)\right|$ is finite since $X_{s}<D$ for all $s<\nu$. Sending $h \rightarrow 0$, we find

$$
\lim _{h \rightarrow 0} \sup _{x \in[0, D]}\left|\tilde{v}_{h}(x)-g(x)\right|=\lim _{h \rightarrow 0} \sup _{x \in[0, D]} \sup _{K \in \mathcal{A}^{0}} \mathbb{P}\left(\theta^{\epsilon,-}<h\right)=0,
$$

by standard SDE bounds. Concluding, the inequality

$$
\lim _{h \downarrow 0} \sup _{x \in[0, D]}\left|v_{1}(\delta-h, x)-v_{1}(\delta, x)\right| \leq \lim _{h \downarrow 0} \sup _{x \in[0, D]}\left|\tilde{v}_{h}(x)-g(x)\right|
$$

and the fact that $g(x)=v_{1}(\delta, x)$ give the desired result.

We skip the proof of the second limit as it could be done similarly.

To prove that $\lim _{x \downarrow 0} v_{1}(\varsigma, x)$ exists, note that for $x \leq y<D$

$$
\begin{aligned}
v_{1}(\varsigma, x) & \geq \sup _{(Z, K) \in \mathcal{A}: \Delta K_{0}=(y-x) /\left(1-\kappa_{1}\right)} \mathbb{E}_{\varsigma, x, 1}\left(\int_{0}^{T} e^{-\rho s}\left(d Z_{s}-d K_{s}\right)\right) \\
& =-(y-x) /\left(1-\kappa_{1}\right)+\sup _{(Z, K)} \mathbb{E}_{\varsigma, y, 1}\left(\int_{0}^{T} e^{-\rho s}\left(d Z_{s}-d K_{s}\right)\right) \\
& =-(y-x) /\left(1-\kappa_{1}\right)+v_{1}(\varsigma, y) .
\end{aligned}
$$

Consequently, the function $y \rightarrow v_{1}\left(\varsigma, y-\frac{y}{1-\kappa_{1}}\right.$ is non increasing on $[0, D]$. Especially, this gives that $v_{1}(\varsigma, 0+)$ exists.

Proposition 2.3. For any $x \in[0, D]$, the function $v_{1}(\cdot, x)$ is non-increasing on $[0, \delta]$ and for any $\varsigma \in[0, \delta]$, the functions $v_{0}$ and $v_{1}(\varsigma, \cdot)$ are non-decreasing respectively on $[0, D]$ and $\mathbb{R}^{+}$.

Proof: For $x \in \mathbb{R}^{+}$, we denote by $X^{x}$ the unique strong solution of equation (2.2) such that $X_{0}^{x}=x$. We also set $T^{x}=\inf \left\{t \geq 0: X_{t}^{x}<0\right\}$ and $\theta_{x}^{D,+}:=\inf \left\{t \geq 0: X_{t}^{x} \geq D\right\}$

Monotonicity of $v_{1}(\varsigma, \cdot)$. Let $\varsigma \in[0, \delta], 0 \leq x \leq y \leq D$ and notice that $\theta_{x}^{D,+} \geq \theta_{y}^{D,+}$. If we 
set $\nu=\inf \left\{t \geq 0: X_{t}^{x}=X_{t}^{y}\right\} \wedge \theta_{y}^{D,+}$, from equation (2.13), we have

$$
\begin{aligned}
v_{1}(\varsigma, x) & =\sup _{(Z, K) \in \mathcal{A}} \mathbb{E}_{\varsigma, x, 1}\left[-\int_{0}^{\nu \wedge T^{x}} e^{-\rho t} d K_{t}+e^{-\rho \nu} v_{1}\left(\Xi_{\nu}, X_{\nu}^{x}\right) \mathbb{1}_{\left\{\nu<T^{x}\right\}}\right] \\
& \leq \sup _{(Z, K) \in \mathcal{A}} \mathbb{E}_{\varsigma, x, 1}\left[-\int_{0}^{\nu \wedge T^{x}} e^{-\rho t} d K_{t}+e^{-\rho \nu} v_{1}\left(\Xi_{\nu}, X_{\nu}^{y}\right) \mathbb{1}_{\left\{\nu<T^{x}\right\}}\right] \\
& \leq v_{1}(\varsigma, y),
\end{aligned}
$$

because $\nu \wedge T^{x} \leq \nu \wedge T^{y}$ and $X_{t}^{x}=X_{t}^{y}$ on $\{t \geq \nu\}$.

Monotonicity of $v_{0}$. It is clear that, as distribution of dividends is allowed for $X$ greater than $D$, we have $v_{0}(y) \geq v_{0}(x)+(y-x) /\left(1+\kappa^{\prime}\right) \geq v_{0}(x)$ for any $D \leq x \leq y$. Now, let $0 \leq x \leq y \leq D$ and set again $\nu=\inf \left\{t \geq 0: X_{t}^{x}=X_{t}^{y}\right\} \wedge \theta_{y}^{D,+}$. From equation (2.12), the prohibiting of dividend distribution below $D$ and the monotonicity of $v_{1}(0, \cdot)$, we have

$$
\begin{aligned}
v_{0}(x)= & \sup _{(Z, K) \in \mathcal{A}} \mathbb{E}_{x, 0}\left[\int_{0}^{\nu \wedge \tau \wedge T^{x}} e^{-\rho s} d\left(Z_{s}-K_{s}\right)+e^{-\rho \nu} v_{0}\left(X_{\nu}^{x}\right) \mathbb{1}_{\left\{\nu<\tau \wedge T^{x}\right\}}\right. \\
& \left.+e^{-\rho \tau} v_{1}\left(0, X_{\tau}^{x}\right) \mathbb{1}_{\left\{\tau \leq \nu \wedge T^{x}\right\}}\right] . \\
\leq & \sup _{K \in \mathcal{A}^{0}} \mathbb{E}_{x, 0}\left[-\int_{0}^{\nu \wedge \tau \wedge T^{x}} e^{-\rho s} d K_{s}+e^{-\rho \nu} v_{0}\left(X_{\nu}^{y}\right) \mathbb{1}_{\{\nu<\tau \wedge T\}}\right. \\
& \left.+e^{-\rho \tau} v_{1}\left(0, X_{\tau}^{y}\right) \mathbb{1}_{\left\{\tau \leq \nu \wedge T^{x}\right\}}\right] . \\
\leq & v_{0}(y) .
\end{aligned}
$$

Monotonicity in duration. Let $x \in[0, D]$ and $0 \leq \varsigma_{1}<\varsigma_{2}<\delta$. Let $\varepsilon>0$. By re-writing the dynamic programming principle (Corollary 2.1), we know that there exists $K^{*} \in \mathcal{A}^{0}$ such that, if we set $\theta=\theta^{D,+} \wedge T$, (associated to the pair $(\Xi, X)$ started at $\Xi_{0}=\varsigma_{2}$ and $X_{0}=x$ ) then we have

$$
\begin{aligned}
v_{1}\left(\varsigma_{2}, x\right) \leq & \mathbb{E}_{\varsigma_{2}, x, 1}\left[\left(-\int_{0}^{\theta} e^{-\rho t} d K_{t}^{*}+e^{-\rho \theta^{D,+}} v_{0}(D)\right) \mathbb{1}_{\left\{\theta<\delta-\varsigma_{2}\right\}}\right] \\
& +\mathbb{E}_{\varsigma_{2}, x, 1}\left[\left(-\int_{0}^{\left(\delta-\varsigma_{2}\right)^{-}} e^{-\rho t} d K_{t}^{*}+e^{-\rho\left(\delta-\varsigma_{2}\right)} g\left(X_{\delta-\varsigma_{2}}\right)\right) \mathbb{1}_{\left\{\theta=\delta-\varsigma_{2}\right\}}\right]+\varepsilon
\end{aligned}
$$

If we apply the same strategy, up to time $\delta-\varsigma_{2}$, to the pair $(\Xi, X)$ starting from the state $\left(\varsigma_{1}, x\right)$ and then issue capital up to $D$ at time $\delta-\varsigma_{2}$, we obtain

$$
\begin{aligned}
v_{1}\left(\varsigma_{1}, x\right) \geq & \mathbb{E}_{\varsigma_{2}, x, 1}\left[\left(-\int_{0}^{\theta} e^{-\rho t} d K_{t}^{*}+e^{-\rho \theta^{D,+}} v_{0}(D)\right) \mathbb{1}_{\left\{\theta<\delta-\varsigma_{2}\right\}}\right] \\
& +\mathbb{E}_{\varsigma_{1}, x, 1}\left[\left(-\int_{0}^{\left(\delta-\varsigma_{2}\right)^{-}} e^{-\rho t} d K_{t}^{*}+e^{-\rho\left(\delta-\varsigma_{2}\right)} g\left(X_{\delta-\varsigma_{2}}\right)\right) \mathbb{1}_{\left\{\theta=\delta-\varsigma_{2}\right\}}\right]
\end{aligned}
$$


which is equal to the right side of (2.16) (up to $\varepsilon$ ) because the coefficients $\mu$ and $\sigma$ are time-independent. Therefore, we get

$$
v_{1}\left(\varsigma_{2}, x\right)-v_{1}\left(\varsigma_{1}, x\right) \leq \varepsilon, \quad \text { for any } \varepsilon>0,
$$

which concludes the proof.

Proposition 2.4. The value function $v_{1}$ is continuous on $\mathcal{S}^{1}$, and $v_{0}$ is continuous on $\mathcal{S}^{0}$.

Proof: $v_{0}$ is obviously continuous on $\mathcal{S}^{0}$ because, from Proposition 2.3 and equation 2.12, it satisfies the following inequalities:

$$
-(y-x) /\left(1-\kappa_{0}\right)+v_{0}(y) \leq v_{0}(x) \leq v_{0}(y), \quad \text { for all } x \leq y \in \mathcal{S}^{0} .
$$

We turn to the continuity of $v_{1}$. Once again, by monotonicity (see Proposition 2.3) and inequality $(2.15), v_{1}(\varsigma, \cdot)$ is continuous, uniformly in $\varsigma$. So we only need to prove that for each $x \in(0, D), v_{1}(\cdot, x)$ is continuous on $[0, \delta]$. The right-continuity of $v_{1}(\cdot, x)$ at $\varsigma=\delta$ follows from Proposition 2.2.

Let $x \in[0, D]$ and $0 \leq \varsigma_{1}<\varsigma_{2}<\delta$. Let $\varepsilon>0$. From Proposition (2.3), we have $v_{1}\left(\varsigma_{2}, x\right)-$ $v_{1}\left(\varsigma_{1}, x\right) \leq 0$. By the dynamic programming principle (Corollary 2.1), we know that there exists $K^{*}$ such that, if we set $\theta=\theta^{D,+} \wedge T$ for the pair $(\Xi, X)$ started at $\Xi_{0}=\varsigma_{2}$ and $X_{0}=x$, then we have

$$
\begin{aligned}
v_{1}\left(\varsigma_{1}, x\right) \leq & \mathbb{E}\left[\left(\int_{0}^{\theta}-e^{-\rho t} d K_{t}^{*}+e^{-\rho \theta^{D,+}} v_{0}(D)\right) \mathbb{1}_{\left\{\theta<\delta-\varsigma_{2}\right\}}\right] \\
& +\mathbb{E}\left[\left(\int_{0}^{\left(\delta-\varsigma_{2}\right)^{-}}-e^{-\rho t} d K_{t}^{*}+e^{-\rho\left(\delta-\varsigma_{2}\right)} v_{1}\left(\delta-\left(\varsigma_{2}-\varsigma_{1}\right), X_{\delta-\varsigma_{2}}\right)\right) \mathbb{1}_{\left\{\theta=\delta-\varsigma_{2}\right\}}\right]+\varepsilon
\end{aligned}
$$

From the dynamic programming principle again, we have

$$
\begin{aligned}
v_{1}\left(\varsigma_{2}, x\right) \geq & \mathbb{E}\left[\left(\int_{0}^{\theta}-e^{-\rho t} d K_{t}^{*}+e^{-\rho \theta^{D,+}} v_{0}(D)\right) \mathbb{1}_{\left\{\theta<\delta-\varsigma_{2}\right\}}\right] \\
& +\mathbb{E}\left[\left(\int_{0}^{\delta^{-}} e^{-\rho t} d K_{t}^{*}+e^{-\rho\left(\delta-\varsigma_{2}\right)} g\left(X_{\delta-\varsigma_{2}}\right)\right) \mathbb{1}_{\left\{\theta=\delta-\varsigma_{2}\right\}}\right]
\end{aligned}
$$

Therefore, we get

$$
v_{1}\left(\varsigma_{2}, x\right)-v_{1}\left(\varsigma_{1}, x\right) \geq \mathbb{E}\left[e^{-\rho\left(\delta-\varsigma_{2}\right)}\left(g\left(X_{\delta-\varsigma_{2}}\right)-v_{1}\left(\delta-\left(\varsigma_{2}-\varsigma_{1}\right), X_{\delta-\varsigma_{2}}\right)\right) \mathbb{1}_{\left\{\theta=\delta-\varsigma_{2}\right\}}\right] .
$$

As in the proof of Proposition 2.2, we can prove that for $\varsigma_{1}$ going to $\varsigma_{2}, \sup _{x \in[0, D]} \mid g(x)-$ $v_{1}\left(\delta-\left(\varsigma_{2}-\varsigma_{1}\right), x\right) \mid \rightarrow 0$, so that $\left|v_{1}\left(\varsigma_{2}, x\right)-v_{1}\left(\varsigma_{1}, x\right)\right|$ converges to 0 as $\left|\varsigma_{2}-\varsigma_{1}\right| \rightarrow 0$. 


\section{Viscosity solution of variational inequalities}

\subsection{Variational inequalities}

In the next section, the value functions $v_{i}$ are shown to satisfy, in the viscosity sense, the following system of variational inequalities, associated to the Dynamic Programming Principle presented in the previous section.

$$
\begin{gathered}
0=\min \left(\rho v_{0}-\mathcal{L} v_{0}-\mathcal{J}\left(v_{0}, v_{1}\right), \mathbb{1}_{[0, D)}+\mathbb{1}_{[D,+\infty)}\left[v_{0}^{\prime}-\frac{1}{1+\kappa^{\prime}}\right], \frac{1}{1-\kappa_{0}}-v_{0}^{\prime}\right) \text { on } \stackrel{\mathcal{S}}{0}^{0} \\
0=\min \left(\rho v_{1}-\mathcal{L} v_{1}-\frac{\partial v_{1}}{\partial \varsigma}, \frac{1}{1-\kappa_{1}}-\frac{\partial v_{1}}{\partial x}\right) \quad \text { on } \stackrel{\circ}{\mathcal{S}}^{1} \\
0=\min \left(v_{1}, \frac{1}{1-\kappa_{1}}-\frac{\partial v_{1}}{\partial x}\right) \quad \text { on }[0, \delta) \times\{0\} \\
0=\min \left(v_{0}(0), \frac{1}{1-\kappa_{0}}-v_{0}^{\prime}(0)\right)
\end{gathered}
$$

where $\mathcal{S}^{i}$ is the interior of $\mathcal{S}^{i}$ and we have set

$$
\mathcal{L} v=\mu(x) \frac{\partial v}{\partial x}+\frac{1}{2} \sigma^{2}(x) \frac{\partial^{2} v}{\partial x^{2}} \quad \text { and } \quad \mathcal{J}(v, w)=\lambda \mathbb{1}_{[0, D]}[w(\cdot, 0)-v] .
$$

Recall that we have set

$$
g(x)=\max \left(v_{0}(D)-(D-x) /\left(1-\kappa_{1}\right), 0\right) \quad \text { on }[0, D]
$$

The value functions $v_{i}$ satisfy the following boundary conditions:

$$
\begin{aligned}
v_{1}(\varsigma, D) & =v_{0}(D) \text { for } \varsigma \in(0, \delta] \\
v_{1}(\delta, x) & =g(x) \text { for } x \in[0, D] .
\end{aligned}
$$

\subsection{Viscosity property and comparison theorem}

We start with proving that $\left(v_{0}, v_{1}\right)$ is a viscosity solution to the previous system of variational inequalities.

Proposition 3.5. The value function $v:=\left(v_{0}, v_{1}\right)$ is a viscosity solution of (3.17)-(3.22).

Proof: The proof of Proposition 3.5 is postponed in the Appendix.

We now turn the uniqueness property. 
Theorem 3.1 (Comparison Theorem). Let $u_{i}(i=0,1)$ be a nonnegative viscosity subsolution of 3.17-3.20, satisfying 3.21-3.22, and $w_{i}(i=0,1)$ a nonnegative viscosity supersolution of 3.17-3.20, satisfying 3.21-3.22. Then, $u_{i} \leq w_{i}(i=0,1)$ on $\mathcal{S}$.

Proof: The proof of Theorem 3.1 is postponed in the Appendix.

\section{Optimal strategies description}

In this section, we describe the following regions of the state space:

$$
\begin{aligned}
\mathcal{K}^{0} & :=\left\{z \in \mathcal{S}^{0}: v_{0}^{\prime}\left(z^{+}\right)=\frac{1}{1-\kappa_{0}}\right\} \\
\mathcal{K}_{\varsigma}^{1} & :=\left\{z \in[0, D]: \frac{\partial v_{1}}{\partial x}\left(\varsigma, z^{+}\right)=\frac{1}{1-\kappa_{1}}\right\}, \quad \mathcal{K}^{1}=\bigcup_{\varsigma \in[0, \delta]}\left(\{\varsigma\} \times \mathcal{K}_{\varsigma}^{1}\right) \\
\mathcal{D} & :=\left\{z \in[D,+\infty): v_{0}^{\prime}\left(z^{-}\right)=\frac{1}{1+\kappa^{\prime}}\right\} \\
\mathcal{C}^{1} & :=\mathcal{S}^{1} \backslash \mathcal{K}^{1} \\
\mathcal{C}^{0} & :=\left(\mathcal{K}^{0} \cup \mathcal{D}\right)^{c}
\end{aligned}
$$

for all $\varsigma \in[0, \delta]$. The set $\mathcal{K}^{i}$ represents the region where it is optimal to inject capital in $\mathcal{S}^{i}$, and $\mathcal{D}$ is the region where it is optimal to payout dividends in $\mathcal{S}^{0}$. The set $\mathcal{C}^{i}$ is the continuation region, where it is neither optimal to inject capital nor payout dividends.

In this section, we turn to the complete resolution of our problem, i.e. obtaining a complete description of the continuation, dividend and capital injection regions enabling us to fully describe the optimal policies. To do so, we shall consider the case in which $\mu$ and $\sigma$ are constant. This complete qualitative description of different regions may only be obtained by introducing an optimal stopping time on a related reflected diffusion process. Solving this optimal stopping-time problem allows us to characterize the optimal issuance of capital policy during the grace period.

\subsection{Optimal strategies during default}

We first recall that as part of the debt covenants, the firm is not allowed to pay out dividends when its debt-to-assets ratio is greater than one, which is the case when the firm is declared in default.

We now begin by recalling the function $g$ and introducing the following function $h$ defined on $\mathbb{R}^{+}$:

$$
g(x)=\left(v_{0}(D)-\frac{1}{1-\kappa_{1}}(D-x)\right)^{+} \quad \text { and } \quad h(x):=\frac{1}{1-\kappa_{1}}\left(x+\frac{\mu}{\rho}\right) .
$$


We also define the following threshold:

$$
\underline{x}_{1}^{*}:=\left(1-\kappa_{1}\right)(h(D)-g(D))=D+\frac{\mu}{\rho}-\left(1-\kappa_{1}\right) v_{0}(D) .
$$

Proposition 4.6. Assume that $\underline{x}_{1}^{*} \leq 0$ then we have $v_{1}(\varsigma, x)=g(x)$ on $\mathcal{S}^{1}$ and $\mathcal{K}^{1}=\mathcal{S}^{1}$.

Proof: The pair $v_{1}=g$ and $v_{0}$ is a viscosity solution of (3.18)-(3.22).

Throughout the rest of this section we shall therefore assume that

$$
\underline{x}_{1}^{*}>0 \text { i.e. } v_{0}(D)<h(D)=\frac{1}{1-\kappa_{1}}\left(D+\frac{\mu}{\rho}\right) .
$$

In this case, $h$ is an upper bound for $v_{1}$ :

Lemma 4.1. For all $(\varsigma, x) \in \mathcal{S}^{1}$, we have $v_{1}(\varsigma, x) \leq h(x)$.

Proof: It is easy to check that the function $h$ is a supersolution of (3.18)-(3.19) with (3.21)$(3.22)$.

Lemma 4.2. For all $\varsigma \in[0, \delta]$, we define $x_{1}^{*}(\varsigma)$ as the smallest solution of the equation

$$
v_{1}(\varsigma, x)=g(x)=\left(v_{0}(D)-\frac{D-x}{1-\kappa_{1}}\right)^{+} \quad \text { on }[0, D] \text {. }
$$

We have the following results:

i) We have $\left(x_{1}^{*}(\varsigma), D\right] \subset \mathcal{K}_{\varsigma}^{1}$ and

$$
x_{1}^{*}(\varsigma)=\sup \left\{x \in[0, D): \frac{\partial v_{1}}{\partial x}\left(\varsigma, x^{+}\right)<\frac{1}{1-\kappa_{1}}\right\} .
$$

ii) For all $\varsigma \in[0, \delta), x_{1}^{*}(\varsigma) \geq \min \left(\underline{x}_{1}^{*}, D\right)$.

iii) The function $\varsigma \rightarrow x_{1}^{*}(\varsigma)$ is non increasing on $[0, \delta]$.

Remark 4.2. Lemma 4.2, points i) and ii) clearly state that when in default, at any given time $\varsigma$ before $\delta$, there exists a threshold $x_{1}^{*}(\varsigma)$ for the assets value above which, it is optimal to inject capital to move the firm's assets value up to $D$ and get out of default.

In Point iii), the threshold $x_{1}^{*}(\varsigma)$ is proved to be non increasing in $\varsigma$. Economically, it means that the more time the firm has before the end of the grace period, the more patient it should be. In other words, when it is still far from the end of the grace period $\delta$, it is optimal to wait until the firm's assets value is close enough to $D$ before injecting more capital to get out of default. 
Proof: As, $v_{1}(\varsigma, D)=v_{0}(D)$, there is in fact a smallest solution $x_{1}^{*}(\varsigma)$ to $(4.25)$.

We first prove assertion $i)$. As the function $z \rightarrow v_{1}(\varsigma, z)$ is increasing, for any $y \in\left[x_{1}^{*}(\varsigma), D\right]$ we have

$$
g(y) \leq v_{1}(\varsigma, y)=v_{1}\left(\varsigma, x_{1}^{*}(\varsigma)\right)+\int_{x_{1}^{*}(\varsigma)}^{y} \frac{\partial v_{1}}{\partial x}(\varsigma, z) d z \leq v_{1}\left(\varsigma, x_{1}^{*}(\varsigma)\right)+\frac{y-x_{1}^{*}(\varsigma)}{1-\kappa_{1}}=g(y) .
$$

Hence, $v_{1}(\varsigma, y)=g(y)$ on $\left[x_{1}^{*}(\varsigma), D\right]$ and that proves assertion $\left.i\right)$.

Recall that $\underline{x}_{1}^{*}>0$. By contradiction, assume that there exists $\varsigma \in[0, \delta)$, such that

$$
x_{1}^{*}(\varsigma)<\underline{y}_{1}^{*}:=\min \left(\underline{x}_{1}^{*}, D\right) .
$$

We notice that

$$
v_{1}\left(\varsigma, \underline{y}_{1}^{*}\right) \geq v_{0}(D)-\frac{D-\underline{y}_{1}^{*}}{1-\kappa_{1}} \geq \min \left(v_{0}(D), \frac{\mu}{\rho\left(1-\kappa_{1}\right)}\right)>0 .
$$

Therefore, we deduce from the monotonicity of $v_{1}$ with respect to $\varsigma$ that there exists $\eta>0$ such that

$$
v_{1}(\varsigma, x)=g(x)>0 \quad \text { on }(\varsigma, \varsigma+\eta) \times\left(\underline{y}_{1}^{*}-\eta, \underline{y}_{1}^{*}\right) .
$$

The above leads to a contradiction as, on $(\varsigma, \varsigma+\eta) \times\left(\underline{y}_{1}^{*}-\eta, \underline{y}_{1}^{*}\right)$, we obtain

$$
0 \leq-\mathcal{L} v_{1}-\frac{\partial v_{1}}{\partial \varsigma}=\frac{\rho}{1-\kappa_{1}}\left(x-\underline{x}_{1}^{*}\right)<0 \text {. }
$$

Consequently, we have shown that $\underline{x}_{1}^{*}(\varsigma) \geq \underline{y}_{1}^{*}=\min \left(\underline{x}_{1}^{*}, D\right)$.

Finally we prove that the function $\varsigma \rightarrow x_{1}^{*}(\varsigma)$ is non increasing on $[0, \delta]$. Let $0 \leq \varsigma_{0}<\varsigma_{1}<\delta$ and define the following function on $\mathcal{S}^{1}$

$$
V(\varsigma, x):=\left\{\begin{array}{cc}
v_{1}(\varsigma, x) & \text { if } \varsigma \leq \varsigma_{0} \text { or } x \leq x_{1}^{*}\left(\varsigma_{0}\right) \\
v_{0}(D)-\frac{1}{1-\kappa_{1}}(D-x) & \text { if } \varsigma>\varsigma_{0} \text { and } x>x_{1}^{*}\left(\varsigma_{0}\right)
\end{array}\right.
$$

We can check that $V$ is a continuous solution of equations (3.18)-(3.22) on $\mathcal{S}^{1}$ so $V=v_{1}$ and $x_{1}^{*}\left(\varsigma_{0}\right) \leq x_{1}^{*}\left(\varsigma_{1}\right)$.

Remark 4.3. On one hand, we have shown that, for any $\varsigma \in[0, \delta)$,

$$
x_{1}^{*}(\varsigma) \geq \min \left(\underline{x}_{1}^{*}, D\right) .
$$

On the other hand, it is easy to check that

$$
x_{1}^{*}(\delta)=\left(D-\left(1-\kappa_{1}\right) v_{0}(D)\right)^{+}<\underline{x}_{1}^{*}
$$


Hence we may have a jump in the optimal threshold $\varsigma \rightarrow x_{1}^{*}(\varsigma)$ at time $\delta$.

Remark 4.4. If $D<\underline{x}_{1}^{*}$ then $x_{1}^{*}(\varsigma)=D$ for any $\varsigma \in[0, \delta)$.

The following lemma is a direct consequence of the monotonicity of $v(\cdot, 0)$ :

Lemma 4.3. There exists a threshold $\varsigma^{*} \in[0, \delta]$ such that

$$
v_{1}(\varsigma, 0)>0 \quad \text { for } 0 \leq \varsigma<\varsigma^{*} \quad \text { and } \quad v_{1}(\varsigma, 0)=0 \quad \text { for } \varsigma^{*} \leq \varsigma<\delta \text {. }
$$

In order to give a complete description of $\mathcal{K}^{1}$, we will prove that the value function $v_{1}$ corresponds to an optimal stopping problem.

Due to the previous lemma, we can define the following auxiliary optimal stopping problem. For $(\varsigma, x) \in[0, \delta) \times[0, D]$, we first introduce a pair of processes $\left(X^{*}, K^{*}\right)$ with the following dynamic:

$$
d X_{t}^{*}:=\mu d t+\sigma d W_{t}+d K_{t}^{*}, \quad X_{0^{-}}^{*}=x, K_{0^{-}}^{*}=0 .
$$

where $X_{t}^{*} \geq 0$ for $t \in\left[\varsigma, \varsigma^{*}\right]$ and $K^{*}$ is a non-decreasing process which satisfies

$$
\int_{0}^{t} \mathbb{1}_{\left\{X_{s}^{*}=0\right\}} d K_{s}^{*}=K_{t}^{*}, \quad \text { for all } 0 \leq t \leq\left(\varsigma^{*}-\varsigma\right)^{+}, \text {and } d K_{t}^{*}=0, \text { for }\left(\varsigma^{*}-\varsigma\right)^{+} \leq t \leq \delta-\varsigma \text {. }
$$

We now define the following optimal stopping problem:

$$
w(\varsigma, x):=\sup _{\nu \in \mathcal{T}_{0, \delta-\varsigma}} \mathbb{E}\left[e^{-\rho \nu \wedge \theta} g\left(X_{\nu \wedge \theta}^{*}\right)-\frac{1}{1-\kappa_{1}} \int_{0}^{\nu \wedge \theta \wedge\left(\varsigma^{*}-\varsigma\right)^{+}} e^{-\rho s} d K_{s}^{*}\right]
$$

where we have set $\theta:=\theta^{*, D,+} \wedge \theta^{*, 0,-}$ with

$$
\theta^{*, D,+}:=\inf \left\{s \geq 0: X_{s}^{*} \geq D\right\} \quad \text { and } \quad \theta^{*, 0,-}:=\inf \left\{s \geq \varsigma^{*}: X_{s}^{*} \leq 0\right\}
$$

We now give the following theorem which allows to fully describe the capital issuance region $\mathcal{K}^{1}$ when the firm is in default. The corresponding continuation region $\mathcal{C}^{1}$ may equally be obtained.

Theorem 4.2. We have $v_{1}=w$ and

$$
\mathcal{K}^{1}:=\left[0, \varsigma^{*}\right) \times\{0\} \cup\left\{(\varsigma, x) \in \mathcal{S}^{1}: x \geq x_{1}^{*}(\varsigma)\right\}
$$

Remark 4.5. Theorem 4.2 states that the capital issuance region includes two disconnected domains.

- The first domain which corresponds to $\mathcal{K}_{1}^{1}:=\left\{(\varsigma, x) \in \mathcal{S}^{1}: x \geq x_{1}^{*}(\varsigma)\right\}$ is as described in Lemma 4.2 and Remark 4.2. When in that domain, it is optimal for the firm to increase the capital by $D-x_{1}^{*}(\varsigma)$, net of issuance cost. 
- The second domain is defined by $\mathcal{K}_{2}^{1}:=\left[0, \varsigma^{*}\right) \times\{0\}$. While in default and the firm's asset value goes to zero, if there is enough time, i.e. $\varsigma<\varsigma^{*}$, it is still optimal to inject capital to keep the firm afloat, i.e. to keep the firm's assets value just above zero and avoid bankruptcy. The assets value process may evolve favorably and reach $\mathcal{K}_{1}^{1}$. However, at $\varsigma \geq \varsigma^{*}$, there is not enough to reach $\mathcal{K}_{1}^{1}$, it is therefore optimal to let the firm go bankrupt.

Proof: We first prove that $w \leq v_{1}$. Let $(\varsigma, x) \in \stackrel{\circ}{\mathcal{S}}^{1}$. We define the following process

$$
K_{t}=K_{t \wedge \tau^{*}}^{*}+\left(D-x_{1}^{*}\left(\varsigma+\tau^{*}\right)\right) \mathbb{1}_{\left\{t \geq \tau^{*}\right\}},
$$

where we have set

$$
\tau^{*}:=\inf \left\{t \geq 0: X_{t}^{*} \geq x_{1}^{*}(\varsigma+t)\right\} .
$$

We can check that $K \in \mathcal{A}^{0}$ and therefore, the dynamic programming principle leads to:

$$
v_{1}(\varsigma, x) \geq \mathbb{E}_{\varsigma, x, 1}\left[e^{-\rho T \wedge \theta^{D,+}} v_{1}\left(T \wedge \theta^{D,+}, X_{T \wedge \theta^{D,+}}^{*}\right)-\int_{0}^{T \wedge \theta^{D,+}} e^{-\rho s} d K_{s}\right]=w(\varsigma, x) .
$$

In a second step, we prove that $w \geq v_{1}$. By construction, it is easy to see that $w$ satisfies (3.21)-(3.22). To prove $w \geq v_{1}$, we can therefore show that $w$ is a supersolution of the HJB equation (3.18)-(3.19). We first prove that

$$
\frac{\partial w}{\partial x} \leq \frac{1}{1-\kappa_{1}}
$$

in the viscosity sense. Let $(\varsigma, x) \in[0, \delta) \times[0, D)$ and $h>0$ such that $x+h \leq D$. Let $\psi \in \mathcal{C}^{1,2}\left(\stackrel{\mathcal{S}}{ }^{1}\right)$ such that $\psi_{1} \leq w$ and $\psi_{1}(\varsigma, x)=w(\varsigma, x)$. We define the pair of processes $\left(X^{*, h}, K^{*, h}\right)$ with the following dynamic:

$$
d X_{t}^{*, h}:=\mu d t+\sigma d W_{t}+\mathbb{1}_{\left\{t \leq \varsigma^{*}\right\}} d K_{t}^{*, h}, \quad X_{0^{-}}^{*, h}=x+h, K_{0^{-}}^{*, h}=0 .
$$

where $X^{*, h} \geq 0$ and $K^{*, h}$ is a non-decreasing process which satisfies

$$
\int_{0}^{t} \mathbb{1}_{\left\{X_{s}^{*, h}=0\right\}} d K_{s}^{*, h}=K_{t}^{*, h}, \quad \text { for all } 0 \leq t \leq \delta .
$$

From the uniqueness of solutions of the stochastic equation (4.31), we have $X^{*, h} \geq X^{*}$. Let $\theta^{h}:=\theta_{h}^{*, D,+} \wedge \theta_{h}^{*, 0,-}$ with

$$
\theta_{h}^{*, D,+}:=\inf \left\{s \geq 0: X_{s}^{*, h} \geq D\right\} \quad \text { and } \quad \theta_{h}^{*, 0,-}:=\inf \left\{s \geq \varsigma^{*}: X_{s}^{*, h} \leq 0\right\} .
$$

If we set $\theta_{h}^{0,-}:=\inf \left\{t \geq 0: x+h+\mu t+\sigma W_{t}=0\right\}$, we have

$$
K_{t \wedge\left(\varsigma^{*}-\varsigma\right)^{+}}^{*}=K_{t \wedge\left(\varsigma^{*}-\varsigma\right)^{+}}^{*}-K_{t \wedge \theta_{h}^{0,-} \wedge\left(\varsigma^{*}-\varsigma\right)^{+}}^{*}
$$


for $t \leq \delta-\varsigma$, and

$$
X_{t}^{*, h}-X_{t}^{*}=h-K_{t \wedge \theta_{h}^{0,-} \wedge\left(\varsigma^{*}-\varsigma\right)^{+}}^{*}
$$

for $t \leq \theta^{*, 0,-}$.

Let $\varepsilon>0$. There exists $\nu^{h} \in \mathcal{T}_{0, \delta-\varsigma}$ such that:

$$
\begin{aligned}
w(\varsigma, x+h) \leq \mathbb{E}\left[e^{-\rho \theta^{*, 0,-}} w\left(X_{\theta^{*, 0,-}}^{*, h}\right) \mathbb{1}_{\left\{\nu^{h} \wedge \theta^{h}>\theta^{*, 0,-}\right\}}+e^{-\rho \nu^{h} \wedge \theta^{h}} g\left(X_{\nu^{h} \wedge \theta^{h}}^{*, h}\right) \mathbb{1}_{\left\{\nu^{h} \wedge \theta^{h} \leq \theta^{*, 0,-}\right\}}\right. \\
\left.-\frac{1}{1-\kappa_{1}} \int_{0}^{\nu^{h} \wedge \theta^{h} \wedge \theta^{*, 0,-} \wedge\left(\varsigma^{*}-\varsigma\right)^{+}} e^{-\rho s} d K_{s}^{*, h}\right]+\varepsilon
\end{aligned}
$$

Moreover, it follows from the definition of $w$, that

$$
w(\varsigma, x) \geq \mathbb{E}\left[e^{-\rho \nu^{h} \wedge \theta^{h}} g\left(X_{\nu^{h} \wedge \theta^{h}}^{*}\right) \mathbb{1}_{\left\{\nu^{h} \wedge \theta^{h} \leq \theta^{*, 0,-}\right\}}-\frac{1}{1-\kappa_{1}} \int_{0}^{\nu^{h} \wedge \theta^{h} \wedge \theta^{*, 0,-} \wedge\left(\varsigma^{*}-\varsigma\right)^{+}} e^{-\rho s} d K_{s}^{*}\right] .
$$

Therefore, we get

$$
\begin{aligned}
w(\varsigma, x+h)-w(\varsigma, x) \leq & \mathbb{E}\left[e^{-\rho \theta^{*, 0,-}} v_{1}\left(\theta^{*, 0,-}, X_{\theta^{*, 0,-}}^{*, h}\right) \mathbb{1}_{\left\{\nu^{h} \wedge \theta^{h} \geq \theta^{*, 0,-}\right\}}\right. \\
& \left.+e^{-\rho \nu^{h} \wedge \theta^{h}}\left(g\left(X_{\nu^{h} \wedge \theta^{h}}^{*, h}\right)-g\left(X_{\nu^{h} \wedge \theta^{h}}^{*}\right)\right) \mathbb{1}_{\left\{\nu^{h} \wedge \theta^{h} \leq \theta^{*, 0,-}\right\}}\right] \\
& +\frac{1}{1-\kappa_{1}} \mathbb{E}\left[\int_{0}^{\nu^{h} \wedge \theta^{h} \wedge \theta^{*, 0,-} \wedge\left(\varsigma^{*}-\varsigma\right)^{+}} e^{-\rho s} d K_{s}^{*}\right]+\varepsilon,
\end{aligned}
$$

since $v_{1} \geq w$. As, for all $\left(y, y^{\prime}\right) \in[0, D]^{2}, g(y)-g\left(y^{\prime}\right) \leq\left(y-y^{\prime}\right) /\left(1-\kappa_{1}\right)$, we get

$$
\begin{aligned}
\mathbb{E}\left[e ^ { - \rho \nu ^ { h } \wedge \theta ^ { h } } \left(g\left(X_{\nu^{h} \wedge \theta^{h}}^{*, h}\right)\right.\right. & \left.\left.-g\left(X_{\nu^{h} \wedge \theta^{h}}^{*}\right)\right) \mathbb{1}_{\left\{\nu^{h} \wedge \theta^{h} \leq \theta^{*, 0,-}\right\}}\right] \\
& \leq \frac{1}{1-\kappa_{1}} \mathbb{E}\left[e^{-\rho \nu^{h} \wedge \theta^{h}}\left(X_{\nu^{h} \wedge \theta^{h}}^{*, h}-X_{\nu^{h} \wedge \theta^{h}}^{*}\right) \mathbb{1}_{\left\{\nu^{h} \wedge \theta^{h} \leq \theta^{*, 0,-}\right\}}\right] \\
& \leq \frac{1}{1-\kappa_{1}} \mathbb{E}\left[\left(h-K_{\tau^{h}}^{*}\right) \mathbb{1}_{\left\{\nu^{h} \wedge \theta^{h} \leq \theta^{*, 0,-}\right\}}\right] .
\end{aligned}
$$

where we have set $\tau^{h}:=\nu^{h} \wedge \theta^{h} \wedge \theta^{*, 0,-} \wedge \theta_{h}^{0,-} \wedge\left(\varsigma^{*}-\varsigma\right)^{+}$. Furthermore, from (2.15), we find

$$
\mathbb{E}\left[e^{-\rho \theta^{*, 0,-}} v_{1}\left(\theta^{*, 0,-}, X_{\theta^{*, 0,-}}^{*, h}\right) \mathbb{1}_{\left\{\nu^{h} \wedge \theta^{h} \geq \theta^{*, 0,-}\right\}}\right] \leq \mathbb{E}\left[\frac{h-K_{\tau^{h}}}{1-\kappa_{1}} \mathbb{1}_{\left\{\nu^{h} \wedge \theta^{h} \geq \theta^{*, 0,-}\right\}}\right]
$$

Plugging this in inequality (4.32), using the fact that $\psi \leq w$ and letting $\varepsilon$ go to 0 , we obtain :

$$
\begin{aligned}
\psi(\varsigma, x+h)-\psi(\varsigma, x) & \leq \frac{1}{1-\kappa_{1}} \mathbb{E}\left[h-K_{\tau^{h}}^{*}+\int_{0}^{\tau^{h}} e^{-\rho s} d K_{s}^{*}\right]+\varepsilon \\
& \leq \frac{h}{1-\kappa_{1}}
\end{aligned}
$$


In particular,

$$
\min \left(w, \frac{1}{1-\kappa_{1}}-\frac{\partial \psi}{\partial x}\right) \geq 0 \text { on }[0, \delta) \times\{0\}
$$

since $w \geq 0$.

Now, we turn to the proof of $w$ being a supersolution of $(3.18)$. Let $(\varsigma, x) \in \mathcal{S}^{1}$ and $\psi \in \mathcal{C}^{1,2}\left(\stackrel{\mathcal{S}}{ }^{1}\right)$ such that $\psi \leq w$ and $\psi(\varsigma, x)=w(\varsigma, x)$. We already know that

$$
\frac{\partial \psi}{\partial x}(\varsigma, x) \leq \frac{1}{1-\kappa_{1}} .
$$

Assume that there exists $\eta>0$ such that

$$
\rho \psi(\varsigma, x)-\mathcal{L} \psi(\varsigma, x)<-\eta
$$

Since $\psi \in C^{1,2}\left(\stackrel{\mathcal{S}}{ }^{1}\right)$, we can assume that there exists $\epsilon>0(\epsilon<\min (x, D-x, \varsigma, \delta-\varsigma))$ such that the above inequality is also satisfied for all $(s, z)$ such that $|z-x|^{2}+|s-\varsigma|<\epsilon$. Let $\theta=\inf \left\{t \geq 0:\left|X_{t}^{*}-x\right|^{2}+|t-\varsigma| \geq \epsilon\right\}$.

By Itô's Formula,

$$
\begin{aligned}
\psi(\varsigma, x)= & \mathbb{E}_{\varsigma, x, 1}\left[e^{-\rho \theta} \psi\left(\varsigma+\theta, X_{\theta}^{*}\right)+\int_{0}^{\theta} e^{-\rho t}\left(\rho \psi\left(\varsigma+t, X_{t}^{*}\right)-\mathcal{L} \psi\left(\varsigma+t, X_{t}^{*}\right)\right) d t\right. \\
& \left.-\int_{0}^{\theta} e^{-\rho t} \psi^{\prime}\left(\varsigma+t, X_{t}^{*}\right) d K_{t}^{*}\right] \\
< & \mathbb{E}_{\varsigma, x, 1}\left[e^{-\rho \theta} \psi\left(\varsigma+\theta, X_{\theta}^{*}\right)-\frac{\eta}{\rho}\left(1-e^{-\rho \theta}\right)\right] . \\
\leq & \mathbb{E}_{\varsigma, x, 1}\left[e^{-\rho \theta} w\left(\varsigma+\theta, X_{\theta}^{*}\right)-\frac{\eta}{\rho}\left(1-e^{-\rho \theta}\right)\right]
\end{aligned}
$$

From the DPP, we have

$$
\begin{aligned}
\psi(\varsigma, x)=w(\varsigma, x) & \geq \mathbb{E}_{\varsigma, x, 1}\left[e^{-\rho \theta} w\left(\varsigma+\theta, X_{\theta}^{*}\right)-\frac{1}{1-\kappa_{1}} \int_{0}^{\theta} e^{-\rho t} d K_{t}^{*}\right] \\
& =\mathbb{E}_{\varsigma, x, 1}\left[e^{-\rho \theta} w\left(\varsigma+\theta, X_{\theta}^{*}\right)\right]
\end{aligned}
$$

It leads to a contradiction since $\mathbb{E} e^{-\rho \theta}<1$. Therefore $w$ is a supersolution of the HJB equation (3.18)-(3.19), (3.21)-(3.22), and from the comparison Theorem (3.1) we know that $w \geq v_{1}$.

\subsection{Optimal strategies before default}

We first turn to the complete description of the dividend region, which is a given interval $\mathcal{D}=\left[x_{0}^{d},+\infty\right)$. 


\section{Lemma 4.4. Dividend region}

There exists a threshold $x_{0}^{d} \geq D$ such that $\mathcal{D}=\left[x_{0}^{d},+\infty\right)$. If $v_{0}(D) \geq \frac{\mu}{\left(1+\kappa^{\prime}\right) \rho}$ then $x_{0}^{d}=D$ else $x_{0}^{d}$ is the unique solution in $(D,+\infty)$ of the following equation:

$$
\rho v_{0}\left(x_{0}^{d}\right)=\frac{\mu}{1+\kappa^{\prime}} .
$$

Moreover, on $(D,+\infty), v_{0}$ is twice continuously differentiable.

Proof: First we assume that $v_{0}(D)<\frac{\mu}{\left(1+\kappa^{\prime}\right) \rho}$. As $v_{0}$ is continuous and increasing on $[0,+\infty)$, there exists $x_{0}^{d}>D$ such that

$$
\rho v_{0}\left(x_{0}^{d}\right)=\frac{\mu}{1+\kappa^{\prime}}
$$

Now we define the following function

$$
V(t, x):=\left\{\begin{array}{cc}
v_{0}(x) & \text { if } x \leq x_{0}^{d} \\
\frac{1}{1+\kappa^{\prime}}\left(x-x_{0}^{d}\right)+v_{0}\left(x_{0}^{d}\right) & \text { if } x>x_{0}^{d}
\end{array}\right.
$$

We can easily prove that $V$ is a viscosity solution of equations (3.17) and (3.20) then $V=v_{0}$. If $v_{0}(D) \geq \frac{\mu}{\left(1+\kappa^{\prime}\right) \rho}$, we can show in the same way that $v_{0}(x)=v_{0}(D)+\frac{1}{1+\kappa^{\prime}}(x-D)$ for all $x \geq D$. Hence, $\mathcal{D}=[D,+\infty)$.

We turn to the proof of concavity on $(D,+\infty)$ for the function $v_{0}$. From equation (3.17), we deduce that on $\left(D, x_{0}^{d}\right)$ :

$$
\begin{aligned}
\frac{1}{2} \sigma^{2} v_{0}^{\prime \prime} & \leq \rho v_{0}-\mu v_{0}^{\prime} \\
& \leq \rho v_{0}-\mu \frac{1}{1+\kappa^{\prime}} \\
& \leq 0 .
\end{aligned}
$$

As $v_{0}$ is linear on $\left[x_{0}^{d},+\infty\right), v_{0}$ is concave on $(D,+\infty)$.

\section{Lemma 4.5. Capital issuance out of financial distress region}

We have $\mathcal{K}^{0} \cap(D,+\infty)=\emptyset$

Remark 4.6. The above Lemma 4.5 states that when the firm's assets value is strictly greater than D, it is not optimal to inject additional capital, which is coherent with the results obtained by Lokka and Zervos [16] in the case when $D=0$.

Proof: Assume, by contradiction, that $\mathcal{K}^{0} \cap(D,+\infty) \neq \emptyset$. From the concavity of $v_{0}$ on $(D,+\infty)$, we deduce that there exists $x_{0}>D$ such that $\left(D, x_{0}\right) \subset \mathcal{K}^{0} \cap(D,+\infty)$. We then have

$$
v_{0}(D)=v_{0}(x)-\frac{1}{1-\kappa_{0}}(x-D) \quad \text { on }\left(D, x_{0}\right) .
$$


From equation (3.17), it follows that

$$
0 \leq \rho v_{0}(D)+\frac{1}{1-\kappa_{0}}(\rho(x-D)-\mu)
$$

Moreover, it follows from Proposition 4.4 that $v_{0}(D)<\frac{\mu}{\left(1+\kappa^{\prime}\right) \rho}$ else we should have $v_{0}^{\prime}(x)=$ $\frac{1}{1+\kappa^{\prime}}$ on $(D,+\infty)$. Hence we obtain

$$
0 \leq-\frac{\mu\left(\kappa^{\prime}+\kappa_{0}\right)}{\left(1+\kappa^{\prime}\right)\left(1-\kappa_{0}\right)}+\frac{\rho}{1-\kappa_{0}}(x-D)
$$

It leads to a contradiction when letting $x$ go to $D$.

We now turn to the description of the capital issuance region $\mathcal{K}^{0}$. We will separate several cases depending on the value of $\underline{x}_{1}^{*}$.

Proposition 4.7. We consider the case $\underline{x}_{1}^{*} \leq 0$. then we have

$$
\dot{\mathcal{K}}^{0}=(0, D) \text { and } v_{0}(x)=g_{0}(x):=\left(v_{0}(D)-\frac{D-x}{1-\kappa_{0}}\right)^{+}, \quad \text { for } x \in[0, D] .
$$

Remark 4.7. In Proposition 4.6, when $\underline{x}_{1}^{*} \leq 0$, we have $\mathcal{K}^{1}=\mathcal{S}^{1}$, i.e. $\mathcal{K}_{\varsigma}^{1}=(0, D)$ $\forall \varsigma \in[0, \delta]$. The above Proposition 4.7 states that when the firm's value is below $D$ but still not in default, the optimal strategy is to straightaway inject capital and not take the risk of being declared in default given the higher issuance cost when in default.

Proof: From Proposition 4.6, we know that if $v_{0}(D) \geq h(D)$ then $v_{1}(t, x)=g(x)$ on $\mathcal{S}^{1}$. It follows that the function $g_{0}$ is solution of the HJB equation (3.17) and (3.20). From the comparison Theorem 3.1, we deduce that $v_{0}=g_{0}$ on $[0, D]$

Proposition 4.8. We consider the case $\underline{x}_{1}^{*}>0$. We define $x_{0}^{*}$ as the smallest solution of the equation

$$
v_{0}(x)=g_{0}(x):=\left(v_{0}(D)-\frac{D-x}{1-\kappa_{0}}\right)^{+} \quad \text { on }(0, D] .
$$

We then have $\left[x_{0}^{*}, D\right) \subset \mathcal{K}^{0}$ and $x_{0}^{*}=\sup \left\{x \in[0, D]: v_{0}^{\prime}(x)<\frac{1}{1-\kappa_{0}}\right\}$.

Furthermore, if $\underline{x}_{1}^{*}>D+\frac{\mu}{\rho}\left(\frac{\kappa_{1}-\kappa_{0}}{1-\kappa_{0}}\right)$, then we have $x_{0}^{*}=D$.

Proof:

We assume $\underline{x}_{1}^{*}>0$. Notice that we should have $g_{0}\left(x_{0}^{*}\right)=v_{0}\left(x_{0}^{*}\right)>0$. For any $y \in\left[x_{0}^{*}, D\right]$ we then have

$$
g_{0}(y) \leq v_{0}(y) \leq v_{0}\left(x_{0}^{*}\right)+\frac{y-x_{0}^{*}}{1-\kappa_{0}}=v_{0}(D)-\frac{D-y}{1-\kappa_{0}} \leq g_{0}(y)
$$


Hence, $v_{0}(y)=v_{0}(D)-\frac{D-y}{1-\kappa_{0}}$ on $\left[x_{0}^{*}, D\right]$ and that proves the result.

We now assume $\underline{x}_{1}^{*}>D+\frac{\mu}{\rho}\left(\frac{\kappa_{1}-\kappa_{0}}{1-\kappa_{0}}\right)$, i.e. $\rho v_{0}(D)<\frac{\mu}{1-\kappa_{0}}$.

We can easily show that $x_{0}^{*}=D$. Indeed, if there was an $\varepsilon>0$ such that $(D-\varepsilon, D) \subset \mathcal{K}_{0}$, we would have $v_{0}(x)=g_{0}(x)$ and $H(x):=\rho v_{0}(x)-\mathcal{L} v_{0}(x)-\mathcal{J}\left(v_{0}(x), v_{1}(0, x)\right) \geq 0$ on $(D-\varepsilon, D)$. However it would lead to a contradiction because $H(D)=\rho v_{0}(D)<-\frac{\mu}{1-\kappa_{0}}<0$ and $H$ is continuous.

\section{$5 \quad$ Numerical results}

\subsection{Numerical procedure}

There are two main difficulties in numerically solving the system of variational inequalities by finite difference methods. The first is due to the boundary conditions in $\mathcal{S}^{1}$. However, if $v(D)$ is known, we can use Theorem 4.2 to solve this part of the domain as a stopping time problem with a reflected process at 0 . The second difficulty is the coupling between regions $\mathcal{S}^{0}$ and $\mathcal{S}^{1}$ through the value $v(D)$. Consequently, the numerical procedure we propose consists in a fixed point problem around $v(D)$. Given $v(D)$, each parts of the domain can be solved independently. It then remains to check if the variational inequality is also satisfied in $x=D$.

The procedure is as follows.

First step: For $a>0$, let $v_{1}^{a}$ be the solution of the following variational inequality on $\mathcal{S}^{1}$ :

$$
\begin{array}{rlrl}
\min \left(\rho v-\mathcal{L} v-\frac{\partial v}{\partial \varsigma}, g^{a}-v\right) & =0 & \text { on } \dot{\mathcal{S}}^{1}, \\
\min \left(v, \frac{1}{1-\kappa_{1}}-\frac{\partial v}{\partial x}\right) & =0 & \text { on }[0, \delta) \times\{0\}, \\
v(\delta, x) & =g^{a}(x) \text { for } x \in[0, D],
\end{array}
$$

where $g^{a}(x):=\left(a-\frac{D-x}{1-\kappa_{1}}\right)^{+}$. To solve this system numerically, we can use explicit finite differences to solve the system by comparing at each point in time $\varsigma$ the solutions with the boundary conditions $v_{1}^{a}(\varsigma, 0)=0$ and $\frac{\partial v_{1}^{a}}{\partial x}(\varsigma, 0)=\frac{1}{1-\kappa_{1}}$, and keep the largest solution of the two at each point in time.

Notice that we have

$$
\text { For any } b \geq a, \quad v_{1}^{a} \leq v_{1}^{b}, \quad \text { on } \mathcal{S}^{1} .
$$

Indeed, for $b \geq a$, it is easy to show that $v_{1}^{b}$ is a supersolution of the previous HJB equation and so, greater than the solution $v_{1}^{a}$.

It follows from the Lipschitz property that:

$$
\text { For any } b \geq a, \quad 0 \leq v_{1}^{b}-v_{1}^{a} \leq b-a, \quad \text { on } \mathcal{S}^{1} .
$$


Second step: Compute the solution of the following variational inequality on $[0, D)$ :

$$
\begin{aligned}
\min \left(\rho v-\mathcal{L} v-\mathcal{J}\left(v, v_{1}^{a}\right), \frac{1}{1-\kappa_{0}}-v^{\prime}\right) & =0 \text { on }(0, D) \\
\min \left(v(0), \frac{1}{1-\kappa_{0}}-v^{\prime}(0)\right) & =0 \\
v(D) & =a
\end{aligned}
$$

As before, this can be solved numerically by considering the boundary condition $v(0)=0$ and $v^{\prime}(0)=\frac{1}{1-\kappa_{0}}$, and select the largest of the two. We denote by $v_{0}^{a}$ the solution on $[0, D)$. Once again we have

$$
\text { For any } b \geq a, \quad v_{0}^{a} \leq v_{0}^{b}, \quad \text { on }[0, D] \text {. }
$$

Indeed, for $b \geq a$, it is easy to show that $v_{0}^{b}$ is a supersolution of the previous HJB equation and so, greater than the solution $v_{0}^{a}$.

Now we can use the property (5.42) to prove that, for $b \geq a, v_{0}^{a}+b-a$ is a supersolution of the HJB equation satisfied by $v_{0}^{b}$. We obtain that

$$
\text { For any } b \geq a, \quad 0 \leq v_{0}^{b}-v_{0}^{a} \leq b-a, \quad \text { on }[0, D)
$$

Third step: Compute the solution of the following variational inequality on $(D, \infty)$ :

$$
\begin{aligned}
\min \left(\rho v-\mathcal{L} v, v^{\prime}-\frac{1}{1+\kappa^{\prime}}, \frac{1}{1-\kappa_{0}}-v^{\prime}\right) & =0 \text { on }(D, \infty) \\
v(D) & =a
\end{aligned}
$$

We denote by $v_{0}^{a}$ the solution on $(D, \infty)$. Once again we have

$$
\text { For any } b \geq a, \quad v_{0}^{a} \leq v_{0}^{b}, \quad \text { on } \mathcal{S}^{0} \text {. }
$$

Indeed, for $b \geq a$, it is easy to show that $v_{0}^{b}$ is a supersolution of the previous HJB equation and so, greater than the solution $v_{0}^{a}$.

Now we can use the property (5.42) to prove that, for $b \geq a, v_{0}^{a}+b-a$ is a supersolution of the HJB equation satisfied by $v_{0}^{b}$. We therefore obtain that

$$
\text { For any } b \geq a, \quad 0 \leq v_{0}^{b}-v_{0}^{a} \leq b-a, \quad \text { on } \mathcal{S}^{0} \text {. }
$$

Fourth step: Find the unique value of $a$ that solves the equation $v_{0}^{a}(D)=a$. We have just proved that $a \rightarrow v_{0}^{a}(D)-a$ is a Lipschitz and nonincreasing function. We obviously have $v_{0}^{0}(D) \geq 0$. Now we want to determine $\lim _{a \rightarrow \infty} v_{0}^{a}(D)-a$. Let $a \geq \max \left(\frac{\mu}{\kappa^{\prime} \rho} ; D\right)$ and, on $\mathcal{S}^{0}$, define the function $\psi^{a}$ by

$$
\psi^{a}(x):=\frac{a}{\mu+\rho a}(\rho x+\mu)
$$


With $a$ large enough, we can check that $\frac{\rho a}{\mu+\rho a} \in\left[\frac{1}{1+\kappa^{\prime}}, \frac{1}{1-\kappa_{0}}\right]$. It follows that $\psi^{a}$ is a supersolution of HJB equations (5.39)-(5.48) and then

$$
v_{1}^{a}(\varsigma, x) \leq \psi^{a}(x), \quad \text { on } \mathcal{S}^{1} \quad \text { and } \quad v_{0}^{a}(x) \leq \psi^{a}(x), \quad \text { on } \mathcal{S}^{0} .
$$

Hence, we get

$$
v_{0}^{a}(D)-a \leq-\frac{\rho a}{\mu+\rho a}(a-D) \leq 0
$$

We can conclude that there exist solutions to the equation $v_{0}^{a}(D)=a$ and that they belong to $\left[0, \max \left(\frac{\mu}{\kappa^{\prime} \rho} ; D\right)\right]$. According to the comparison result (see Theorem 3.1), there exists a unique solution that can be found by dichotomy methods.

\subsection{Numerical illustrations}

We present in this section a number of numerical examples that illustrate the above theoretical results. Figure 1 gives an example of a region $\mathcal{S}^{1}$ in which there is no reflection at $x=0$ in $\mathcal{S}^{1}$, but the optimal capital injection happens before $x=D$. The effect of $\kappa_{1}$ on the capital region is illustrated in Figure 2 in which we see that larger values of $\kappa_{1}$ make the region smaller and more to the right.

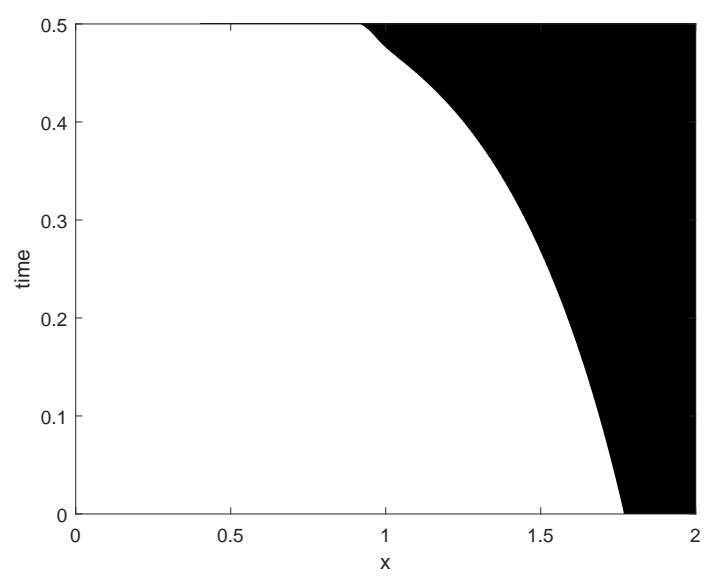

Figure 1: Optimal strategy in $\mathcal{S}^{1}$. Parameters are $D=2, \delta=0.5, \kappa_{0}=\kappa_{1}=\kappa^{\prime}=0.1, \lambda=$ $5, \mu=0.05, \rho=0.1$ and $\sigma=1$. The black area represents the region where it is optimal to inject capital in $\mathcal{S}^{1}$, i.e. $v^{1}=g$.

On the other hand, Figure 3 shows the case where it is only optimal to reflect the process $X$ on the boundary $x=0$ and $\varsigma \in\left[0, \varsigma^{*}\right]$, as defined in Lemma 4.3. For this particular choice of parameters, it is not optimal to inject capital for other values in $\mathcal{S}^{1}$. We notice that for values of $\kappa_{1}=0.07$ or less, the reflection of the process $X$ is for all $\varsigma \in[0, \delta]$. 


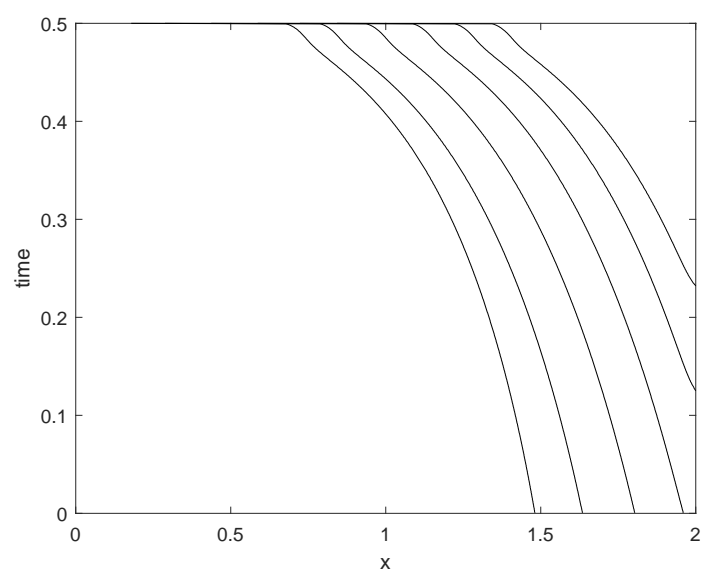

Figure 2: Boundaries of the optimal strategy in $\mathcal{S}^{1}$. Parameters are $D=2, \delta=0.5, \kappa_{0}=$ $\kappa^{\prime}=0.1, \lambda=5, \mu=0.05, \rho=0.1$ and $\sigma=1$. The values of the parameter $\kappa_{1}$ varies from $0,0.05,0.1,0.15,0.2$ to 0.25 , from left to right in the figure.

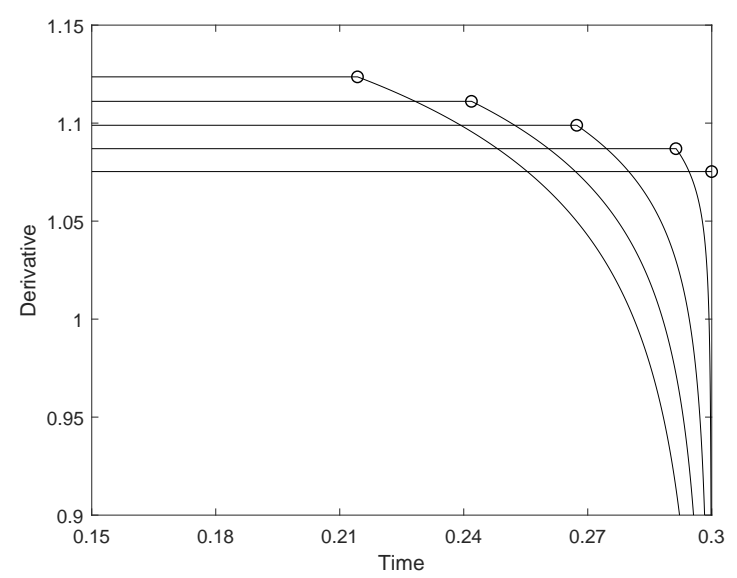

Figure 3: Value of $\frac{\partial v_{1}(\varsigma, 0)}{\partial x}$ for various values of $\kappa_{1}$. Parameters are $D=0.2, \delta=0.3, \kappa_{0}=$ $0.1, \kappa^{\prime}=0.01, \lambda=5, \mu=0.05, \rho=0.1$ and $\sigma=1$. The values of the parameter $\kappa_{1}$ varies from $0.07,0.08,0.09,0.1$ to 0.11 , from bottom to top in the figure. Circles show the value $\varsigma^{*}$ of Lemma 4.3. The largest value of the derivative is $\frac{1}{1-\kappa_{1}}$ for $\varsigma \in\left[0, \varsigma^{*}\right]$.

Figure 4 shows how the value function $v^{0}$ depends on the debt level $D$. The value function at the point $x_{0}^{d}$ starting at which it is optimal to issue dividends is shown to equal $\frac{\mu}{\rho\left(1+\kappa^{\prime}\right)}$, which is independent of $D$ (see Proposition 4.4).

Figures 5 and 6 show the derivative of the function $v^{0}$ on $\mathcal{S}^{0}$ for varying values of $D$. For $D=0.1,0.2$ or 0.3 , (Fig. 5 ) it is optimal to inject capital only at $x=0$. It is never optimal to inject capital for the other values of $D$ considered (Fig. 6). Furthermore, note that the derivative of the function goes below the value $\frac{1}{1+\kappa^{\prime}}=0.909$ for some values of $x$ less than $D$, for $D=0.6,0.8,1$ and 1.5 . This is only possible because it is not permitted to distribute dividends for those values of $x<D$. In other words, the constraint $v_{0}^{\prime}(x) \geq \frac{1}{1+\kappa^{\prime}}$ does not apply. 


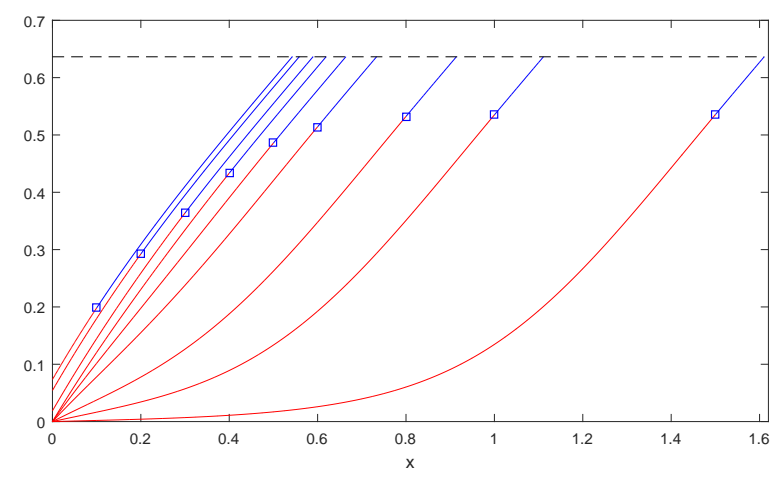

Figure 4: Sensitivity analysis of the value function $v_{0}(x)$ in terms of $D$, taking values in $\{0.1,0.2,0.3,0.4,0.5,0.6,0.8,1,1.5\}$ (from left to right in the graph). Other parameters are fixed to $\delta=0.3, \kappa_{0}=\kappa_{1}=0.25, \kappa^{\prime}=0.1, \lambda=1, \mu=0.07, \rho=0.1$ and $\sigma=0.3$. Blue squares represent the value function at $x=D$. Dashed line is at level $\frac{\mu}{\rho\left(1+\kappa^{\prime}\right)}$, the value at which it is optimal to pay dividends, independent of the value of $D$.

Figures 5 and 6 correspond to sensitivity analysis of the derivative of the value function $v_{0}$ in terms of $D$. Other parameters are fixed to $\delta=0.3, \kappa_{0}=\kappa_{1}=0.25, \kappa^{\prime}=0.1, \lambda=1, \mu=0.07$, $\rho=0.1$ and $\sigma=0.3$. Dashed lines are at levels $\frac{1}{1-\kappa_{0}}=1.3333$ and $\frac{1}{1+\kappa^{\prime}}=0.909$. Blue squares represent the value of the derivative at $x=D$.

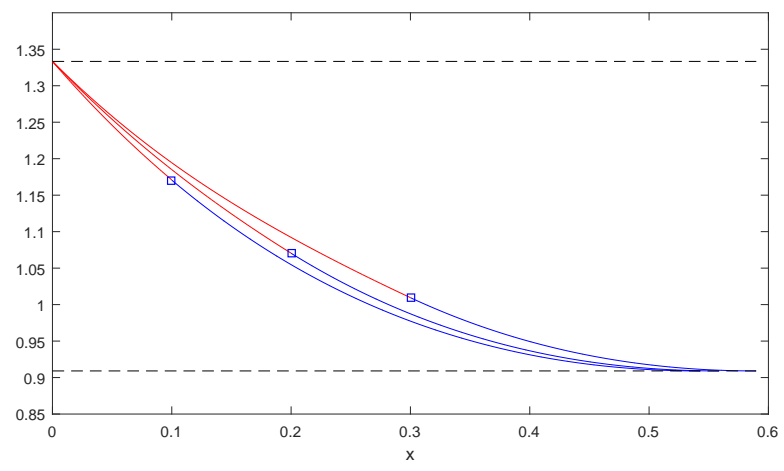

Figure 5: $D$ takes values in $\{0.1,0.2,0.3\}$ from left to right. 


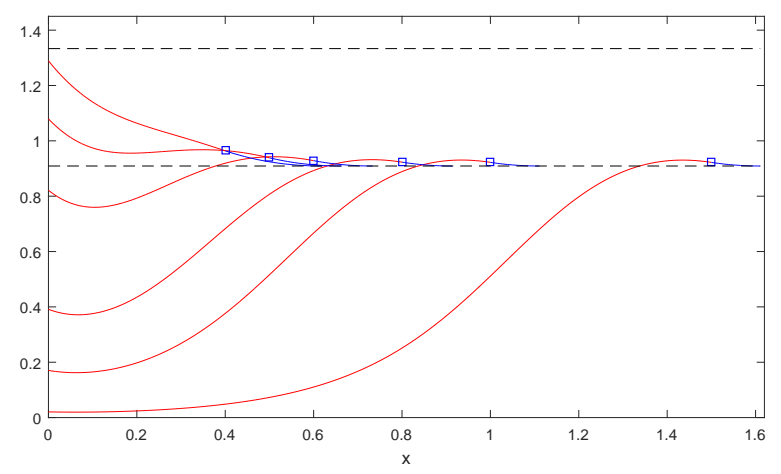

Figure 6: $D$ takes values in $\{0.4,0.5,0.6,0.8,1,1.5\}$ from left to right.

\section{Appendix A : Proof of Proposition 3.5}

Proof: Equations 3.21 and 3.22 have been proved to be satisfied in Proposition 2.2.

The proof of the supersolution property is classical, so we omit it and focus on the subsolution property.

Let $(\varsigma, x, y) \in \stackrel{\mathcal{S}}{1}^{1} \times \dot{\mathcal{S}}^{0}$. Let $\psi_{0} \in C^{2}\left(\dot{\mathcal{S}}^{0}\right)$ and $\psi_{1} \in C^{1,2}\left(\dot{\mathcal{S}}^{1}\right)$ such that $\psi_{1}(\varsigma, x)=v_{1}(\varsigma, x)$, $\psi_{0}(y)=v_{0}(y)$ and $\psi_{i} \geq v_{i}, i=0,1$. We have to prove that $\left(\psi_{0}, \psi_{1}\right)$ satisfies $(3.17)-(3.20)$ with $=$ replaced by $\geq$. Suppose that it is not true:

Case (1) Assume that the inequality coming from (3.17) is not true for some $x>0$. There exist $\eta>0$ and $0<\epsilon<\min (x, 1)$ such that for all $y$ satisfying $|x-y|<\epsilon$,

$$
\begin{array}{r}
\rho \psi_{0}(y)-\mathcal{L} \psi_{0}(y)-\lambda\left(v_{1}(0, y)-\psi_{0}(y)\right)>\eta \text { and }\left(1-\kappa_{0}\right) \psi_{0}^{\prime}(y)<1-\eta \text { if } y<D, \\
\rho \psi_{0}(y)-\mathcal{L} \psi_{0}(y)>\eta,\left(1+\kappa^{\prime}\right) \psi_{0}^{\prime}(y)>1+\eta \text { and }\left(1-\kappa_{0}\right) \psi_{0}^{\prime}(y)<1-\eta \text { otherwise. }
\end{array}
$$

Consider $\varepsilon_{1}=x+\epsilon$ and $\varepsilon_{2}=x-\epsilon$ (as positive constants), and set $\theta=\inf \{s \geq t$ : $\left.\left|X_{s}^{x}-x\right|>\epsilon\right\}$. Let $\xi=\inf \left\{s \geq 0: I_{s}=1\right\}$, and notice that $\left|X_{\theta}-x\right|=\epsilon$ on $\{\theta<\xi\}$, for any $(Z, K) \in \mathcal{A}$. Recall that $d Z_{t}=0$ on $\left\{X_{t}<D\right\}$. Consequently, $\left(1+\kappa^{\prime}\right) \psi_{0}^{\prime}\left(X_{t}\right) d Z_{t} \geq(1+\eta) d Z_{t}$, for all $t \geq 0$. We can therefore show that

$$
\begin{aligned}
& \mathbb{E}_{x, 0}\left[\int_{0}^{\theta \wedge \xi} e^{-\rho t}\left(d Z_{t}-d K_{t}\right)+e^{-\rho \theta} v_{0}\left(X_{\theta}\right) 1_{\{\theta<\xi\}}+e^{-\rho \xi} v_{1}\left(0, X_{\theta}\right) 1_{\{\xi \leq \theta\}}\right] \\
& \leq \mathbb{E}_{x, 0}\left[\int_{0}^{\theta \wedge \xi} e^{-\rho t}\left(d Z_{t}-d K_{t}\right)+e^{-\rho(\theta \wedge \xi)} \psi_{0}\left(X_{\theta \wedge \xi}\right)+\int_{0}^{\theta \wedge \xi} \lambda e^{-\rho t}\left(\psi_{0}\left(X_{t}\right)-v_{1}\left(0, X_{t}\right)\right) 1_{\left\{X_{t}<D\right\}} d t\right] \\
& \leq \psi_{0}(x)-\eta \mathbb{E}_{x, 0}\left[\int_{0}^{\theta \wedge \xi} e^{-\rho t} d t+\int_{0}^{\theta \wedge \xi} e^{-\rho t}\left(d Z_{t}+d K_{t}\right)\right] .
\end{aligned}
$$

By considering $\varphi(y)=C\left((y-x)^{2}-\epsilon^{2}\right)$, for some positive constant $C<\min \left(\frac{1}{2 \epsilon}, \frac{1}{(\rho+\lambda) \epsilon^{2}+2 \bar{\mu} \epsilon+\bar{\sigma}^{2}}\right)$, 
we find that

$$
\begin{aligned}
C \epsilon^{2}= & \mathbb{E}_{x, 0}\left[e^{-\rho \theta} \varphi\left(X_{\theta}\right) 1_{\{\theta \leq \xi\}}-\varphi(x)\right] \\
= & \mathbb{E}_{x, 0}\left[\int_{0}^{\theta \wedge \xi} e^{-\rho t}\left(-\left(\rho+\lambda 1_{\left\{X_{t}<D\right\}}\right) \varphi\left(X_{t}\right)+2 C \mu\left(X_{t}\right)\left(X_{t}-x\right)+C \sigma^{2}\left(X_{t}\right)\right) d t\right. \\
& \left.\quad+\int_{0}^{\theta \wedge \xi} e^{-\rho t} 2 C\left(X_{t}-x\right)\left(\left(1-\kappa_{0}\right) d K_{t}-\left(1+\kappa^{\prime}\right) d Z_{t}\right)\right] \\
\leq & \mathbb{E}_{x, 0}\left[\int_{0}^{\theta \wedge \xi} e^{-\rho t} d t+\int_{0}^{\theta \wedge \xi} e^{-\rho t}\left(d Z_{t}+d K_{t}\right)\right] .
\end{aligned}
$$

By taking a supremum over $(Z, K) \in \mathcal{A}$ in (5.51) and applying the dynamic programming principle (2.12), we find the contradiction:

$$
v_{0}(x) \leq \psi_{0}(x)-\eta C \epsilon^{2}<\psi_{0}(x)=v_{0}(x)
$$

Case (2): Assume that inequality associated to (3.18) is not satisfied.

There exists $\eta>0$ such that

$$
\rho \psi_{1}(\varsigma, x)-\mathcal{L} \psi_{1}(\varsigma, x)>\eta, \text { and }\left(1-\kappa_{1}\right) \frac{\partial \psi_{1}}{\partial x}(\varsigma, x)<1-\eta
$$

Since $\psi_{1} \in C^{1,2}\left(\stackrel{\circ}{\mathcal{S}}^{1}\right)$, we can assume that there exists $\epsilon>0(\epsilon<\min (x, D-x, \varsigma, \delta-\varsigma))$ such that the above inequality is also satisfied for all $(s, z)$ such that $|x-z|^{2}+|s-\varsigma| \leq \epsilon$.

Consider $\varepsilon_{1}(s)=x+\sqrt{\epsilon-(s-\varsigma)}$ and $\varepsilon_{2}(s)=x-\sqrt{\epsilon-(s-\varsigma)}$, for $s<\varsigma+\epsilon, \varepsilon_{1}(s)=\varepsilon_{2}(s)=$ $x$ otherwise. Let $(Z, K) \in \mathcal{A}$. Then, if we set $\theta:=\inf \left\{t \geq 0:\left|X_{t}^{x}-x\right|^{2}+\Xi_{t}-\varsigma>\epsilon\right\}$, we have $\left|X_{\theta}-x\right|^{2}=\epsilon-\theta$, by definition of $\mathcal{A}$. Furthermore, $\theta<T \wedge \theta^{D,+}$ and $\left|X_{t}-x\right|^{2}+\Xi_{t}-\varsigma \leq \epsilon$ for all $t \leq \theta$.

By Itô's Formula,

$$
\begin{aligned}
\psi_{1}(\varsigma, x)=\mathbb{E}_{\varsigma, x, 1} & {\left[e^{-\rho \theta} \psi_{1}\left(\Xi_{\theta}, X_{\theta}\right)+\int_{0}^{\theta} e^{-\rho t}\left(\rho \psi_{1}\left(\Xi_{t}, X_{t}\right)-\mathcal{L} \psi_{1}\left(\Xi_{t}, X_{t}\right)\right) d t\right.} \\
& -\int_{0}^{\theta} e^{-\rho t}\left(1-\kappa_{1}\right) \frac{\partial \psi_{1}}{\partial x}\left(\Xi_{t}, X_{t}\right) d K_{t}^{c} \\
& \left.-\sum_{0 \leq t \leq \theta} e^{-\rho t}\left(\psi_{1}\left(\Xi_{t}, X_{t-}+\left(1-\kappa_{1}\right) \Delta K_{t}\right)-\psi_{1}\left(\Xi_{t}, X_{t-}\right)\right)\right] .
\end{aligned}
$$

Due to $(5.52),\left(1-\kappa_{1}\right) \frac{\partial \psi_{1}}{\partial x}\left(\Xi_{t}, X_{t}\right)<1-\eta$ and $\psi_{1}\left(\Xi_{t}, X_{t-}+\left(1-\kappa_{1}\right) \Delta K_{t}\right)-\psi_{1}\left(\Xi_{t}, X_{t-}\right)<$ $(1-\eta) \Delta K_{t}$. Consequently,

$$
\begin{aligned}
\psi_{1}(\varsigma, x) \geq & \mathbb{E}_{\varsigma, x, 1}\left[-\int_{0}^{\theta} e^{-\rho t} d K_{t}+e^{-\rho \theta} \psi_{1}\left(\Xi_{\theta}, X_{\theta}\right)\right] \\
& +\mathbb{E}_{\varsigma, x, 1}\left[\int_{0}^{\theta} e^{-\rho t}\left(\rho \psi_{1}\left(\Xi_{t}, X_{t}\right)-\mathcal{L} \psi_{1}\left(\Xi_{t}, X_{t}\right)\right) d t+\eta \int_{0}^{\theta} e^{-\rho t} d K_{t}\right]
\end{aligned}
$$


Combining the previous inequality with the fact that $\psi_{1} \geq v_{1}$ and (5.52), we get

$$
v_{1}(\varsigma, x) \geq \mathbb{E}_{\varsigma, x, 1}\left[-\int_{0}^{\theta} e^{-\rho t} d K_{t}+e^{-\rho \theta} v_{1}\left(\Xi_{\theta}, X_{\theta}\right)+\eta \int_{0}^{\theta} e^{-\rho t}\left(d t+d K_{t}\right)\right] \text {. }
$$

Let $\varphi(s, z)=C\left((z-x)^{2}-\epsilon+(s-\varsigma)\right)$, for some positive constant $C<\min \left(\frac{1}{2 \sqrt{\epsilon}}, \frac{1}{\rho \epsilon+2 \bar{\mu} \sqrt{\epsilon}+\bar{\sigma}^{2}+1}\right)$ in which

$$
\bar{\mu}=\sup _{x \in[0, D]} \mu(x), \bar{\sigma}=\sup _{x \in[0, D]} \sigma(x)
$$

By Itô's formula,

$$
\begin{aligned}
C \epsilon= & \mathbb{E}_{\varsigma, x, 1}\left[e^{-\rho \theta} \varphi\left(\Xi_{\theta}, X_{\theta}\right)-\varphi(\varsigma, x)\right] \\
\leq \mathbb{E}_{\varsigma, x, 1} & {\left[\int_{0}^{\theta} e^{-\rho t}\left(-\rho \varphi\left(\Xi_{t}, X_{t}\right)+2 \mu\left(X_{t}\right) C\left(X_{t^{-}}-x\right)+C \sigma^{2}\left(X_{t}\right)+C\right) d t\right] } \\
& \quad+\mathbb{E}_{\varsigma, x, 1}\left[-\int_{0}^{\theta} e^{-\rho t} 2 C\left(X_{t^{-}}-x\right)\left(1-\kappa_{1}\right) d K_{t}\right] \\
\leq & \mathbb{E}_{\varsigma, x, 1}\left[\int_{0}^{\theta} e^{-\rho t} d t+\int_{0}^{\theta} e^{-\rho t} d K_{t}\right] .
\end{aligned}
$$

Taking a supremum over $(Z, K) \in \mathcal{A}$ in (5.53) and applying the dynamic programming principle (2.13), we find the following contradiction:

$$
v_{1}(t, x) \geq v_{1}(t, x)+\eta C \epsilon
$$

Case (3) Assume that inequality coming from (3.19) is not verified for some $(\varsigma, 0) \in[0, \delta] \times$ $\{0\}$ : there exists $\eta$ and $t>0$ such that for all $\epsilon>0,0 \leq x \leq \epsilon$, and $\varsigma \leq s \leq \varsigma+t$

$$
v_{1}(s, x)>\eta, \quad \frac{\partial \psi_{1}}{\partial x}(s, x)<\frac{1}{1-\kappa_{1}}-\eta \text { and } \psi_{1}(s, 0)<\psi_{1}(\varsigma, 0)+C(s-\varsigma),
$$

due to the continuity of $v_{1}$ and $\frac{\partial \psi_{1}}{\partial x}$. Here $C=\sup _{\varsigma \leq s \leq \varsigma+t} \frac{\partial \psi_{1}(s, 0)}{\partial s}$. Assume further that $\epsilon<\min \left(D, v_{1}(\varsigma, 0) / \eta\right)$ and $t<\delta-\varsigma$.

We consider $\theta:=\inf \left\{s \geq 0: X_{s} \geq \varepsilon\right\}$ and deduce from (5.54) and the dynamic programming principle (2.13) that,

$$
\begin{aligned}
v_{1}(\varsigma, 0) & =\sup _{(Z, K) \in \mathcal{A}, Z \equiv 0} \mathbb{E}_{\varsigma, 0,1}\left[-I_{1}+e^{-\rho \theta} v_{1}\left(\Xi_{\theta}, \epsilon\right) \mathbb{1}_{\{\theta<T \wedge t\}}+e^{-\rho t} v_{1}\left(\Xi_{t}, X_{t}\right) 1_{\{t \leq \theta \wedge T\}}\right] \\
& \leq \sup _{K \in \mathcal{A}^{0}} \mathbb{E}_{\varsigma, 0,1}\left[-I_{1}+e^{-\rho \theta} \psi_{1}\left(\Xi_{\theta}, \epsilon\right) \mathbb{1}_{\{\theta<T \wedge t\}}+A_{0} 1_{\{t \leq \theta \wedge T\}}\right]
\end{aligned}
$$

where $A_{0}=\sup _{0 \leq x \leq \epsilon} v_{1}\left(\Xi_{t}, x\right)$, a finite quantity, and $I_{1}$ stands for $\int_{0}^{\theta \wedge T \wedge t} e^{-\rho s} d K_{s}$. We can 
therefore bound the value $v_{1}(\varsigma, 0)$ above by

$$
\begin{aligned}
& \sup _{K \in \mathcal{A}^{0}} \mathbb{E}_{\varsigma, 0,1}\left[-I_{1}+e^{-\rho \theta}\left(\psi_{1}\left(\Xi_{\theta}, 0\right)+\left(\frac{1}{1-\kappa_{1}}-\eta\right) \epsilon\right) \mathbb{1}_{\{\theta<T \wedge t\}}+A_{0} 1_{\{t \leq \theta \wedge T\}}\right] \\
\leq & \psi_{1}(\varsigma, 0)-\eta \epsilon+\sup _{K \in \mathcal{A}^{0}} \mathbb{E}_{\varsigma, 0,1}\left[-I_{1}+e^{-\rho \theta}\left(C \theta+\frac{1}{1-\kappa_{1}} \epsilon\right) \mathbb{1}_{\{\theta<T \wedge t\}}+A_{0} 1_{\{t \leq \theta \wedge T\}}\right] \\
\leq & v_{1}(\varsigma, 0)-\eta \epsilon+\sup _{K \in \mathcal{A}^{0}} \mathbb{E}_{\varsigma, 0,1}\left[-I_{1}+C \theta+e^{-\rho \theta} \frac{1}{1-\kappa_{1}} X_{\theta} \mathbb{1}_{\{\theta<T \wedge t\}}+A_{0} 1_{\{t \leq \theta \wedge T\}}\right] \\
\leq & v_{1}(\varsigma, 0)-\eta \epsilon+\sup _{K \in \mathcal{A}^{0}} \mathbb{E}_{\varsigma, 0,1}\left[C \theta+\int_{0}^{\theta \wedge T \wedge t} e^{-\rho s}\left(\frac{\mu\left(X_{s}\right)-\rho X_{s}}{1-\kappa_{1}}\right) d s+A_{0} 1_{\{t \leq \theta \wedge T\}}\right] \\
\leq & v_{1}(\varsigma, 0)-\eta \epsilon+\left(C+C^{\prime}\right) \sup _{K \in \mathcal{A}^{0}} \mathbb{E}_{\varsigma, 0,1}[\theta]+A_{0} \mathbb{P}(t \leq \theta) \\
\leq & v_{1}(\varsigma, 0)-\eta \epsilon+\frac{\epsilon^{2}\left(C+C^{\prime}+A_{0} / t\right)}{\underline{\sigma}^{2}}
\end{aligned}
$$

where $C^{\prime}=\sup _{x<D}\left(\frac{\mu(x)-\rho x}{1-\kappa_{1}}\right)<\infty$. We find a contradiction by taking $\epsilon$ strictly less than

$$
\frac{\eta \underline{\sigma}^{2}}{\left(C+C^{\prime}+A_{0} / t\right)}
$$

Case (4) Assume that the inequality coming from (3.20) is not verified : there exists $\eta>0$ such that for all $\epsilon>0$

$$
v_{0}(x)>\eta \quad \text { and } \quad \psi_{0}^{\prime}(x)<\frac{1}{1-\kappa_{0}}-\eta \text { for all } 0 \leq x<\epsilon,
$$

due to the continuity of $v_{0}$ and $\psi_{0}^{\prime}$. Assume further that $\epsilon<\min \left(D, v_{0}(0) / \eta\right)$. As before, consider $\theta:=\inf \left\{s \geq 0: X_{s} \geq \varepsilon\right\}, \xi=\inf \left\{s \geq 0: I_{s}=1\right\}$, and notice that $\left|X_{\theta}-x\right|=\epsilon$ on $\{\theta<\xi\}$, and $X_{t}<D$, for all $t<\theta$. We also set $I_{0}:=\int_{0}^{\theta \wedge T \wedge \xi} e^{-\rho t} d K_{t}$ and deduce from (5.55) and the dynamic programming principle (2.13) that

$$
\begin{aligned}
v_{0}(0) & =\sup _{(Z, K) \in \mathcal{A}, Z \equiv 0} \mathbb{E}_{0}\left[-I_{0}+e^{-\rho \theta} v_{0}(\epsilon) \mathbb{1}_{\{\theta<T \wedge \xi\}}+e^{-\rho \xi} v_{1}\left(0, X_{\xi}\right) 1_{\{\xi \leq \theta \wedge T\}}\right] \\
& \leq \sup _{K \in \mathcal{A}^{0}} \mathbb{E}_{0}\left[-I_{0}+e^{-\rho \theta} \psi_{0}(\epsilon) \mathbb{1}_{\{\theta<T \wedge \xi\}}+e^{-\rho \xi} v_{1}\left(0, X_{\xi}\right) 1_{\{\xi \leq \theta \wedge T\}}\right] \\
& \leq \sup _{K \in \mathcal{A}^{0}} \mathbb{E}_{0}\left[-I_{0}+e^{-\rho \theta}\left(v_{0}(0)+\left(\frac{1}{1-\kappa_{0}}-\eta\right) \epsilon\right) \mathbb{1}_{\{\theta<T \wedge \xi\}}+e^{-\rho \xi} v_{1}\left(0, X_{\xi}\right) 1_{\{\xi \leq \theta \wedge T\}}\right] \\
\leq & v_{0}(0)-\eta \epsilon+\sup _{K \in \mathcal{A}^{0}} \mathbb{E}_{0}\left[-I_{1}+e^{-\rho \theta} \frac{1}{1-\kappa_{0}} X_{\theta} \mathbb{1}_{\{\theta<T \wedge \xi\}}+e^{-\rho \xi} v_{1}\left(0, X_{\xi}\right) 1_{\{\xi \leq \theta \wedge T\}}\right] \\
\leq & v_{0}(0)-\eta \epsilon+\sup _{K \in \mathcal{A}^{0}} \mathbb{E}_{0}\left[\int_{0}^{\theta \wedge T \wedge \xi} e^{-\rho t}\left(\frac{\mu\left(X_{t}\right)-\rho X_{t}}{1-\kappa_{0}}+\lambda v_{1}(0, \epsilon)\right) d t\right] \\
\leq & v_{0}(0)-\eta \epsilon+C \sup _{K \in \mathcal{A}^{0}} \mathbb{E}_{0}[\theta],
\end{aligned}
$$


for some $C>0$.

Let $K \in \mathcal{A}^{0}, \check{X}_{t}=\int_{0}^{t} \sigma\left(X_{s}\right) d W_{s}$ and $\check{\theta}=\inf \left\{t \geq 0: \check{X}_{t} \geq \epsilon\right\}$. Since $X \geq \check{X}$, it is clear that $\theta \leq \check{\theta}$. Moreover,

$$
\epsilon^{2}=\mathbb{E}\left[\check{X}_{\check{\theta}}\right]^{2} \geq \underline{\sigma}^{2} \mathbb{E}[\check{\theta}]
$$

where $\underline{\sigma}=\inf _{x<D} \sigma(x)>0$. Combining this result with the above inequalities, we find that $v_{0}(0) \leq v_{0}(0)-\eta \epsilon+C \frac{\epsilon^{2}}{\underline{\sigma}^{2}}$. By taking $\epsilon$ small enough, we find a contradiction.

\section{Appendix B : Proof of Theorem 3.1}

Let $0<\delta<\min \left(\frac{\kappa^{\prime}}{1+\kappa^{\prime}}, \frac{\kappa_{0}}{1-\kappa_{0}}, \frac{\kappa_{1}}{1-\kappa_{1}}\right)$ and $h(x)=A+x$ where $A \geq \max \left(\delta, \frac{1}{\rho}\left(\delta+\sup _{x}(\mu(x)-\right.\right.$ $\rho x)))$. Direct calculation shows that $\rho h-\mathcal{L} h \geq \delta$, and if $0<\gamma<1$, then $w_{i}^{\gamma}:=\gamma h+(1-\gamma) w_{i}$, $(i=0,1)$ is a strict viscosity supersolution of 3.17-3.20:

$$
\begin{aligned}
& \gamma \delta \leq \min \left(\rho w_{0}^{\gamma}-\mathcal{L} w_{0}^{\gamma}-\mathcal{J}\left(w_{0}^{\gamma}, w_{1}^{\gamma}\right), \mathbb{1}_{[0, D)}+\mathbb{1}_{[D,+\infty)}\left[\left(w_{0}^{\gamma}\right)^{\prime}-\frac{1}{1+\kappa^{\prime}}\right]\right. \\
&\left.\frac{1}{1-\kappa_{0}}-\left(w_{0}^{\gamma}\right)^{\prime}\right) \text { on } \mathcal{S}^{0}, \\
& \gamma \delta \leq \min \left(\rho w_{1}^{\gamma}-\mathcal{L} w_{1}^{\gamma}-\frac{\partial w_{1}^{\gamma}}{\partial \varsigma}, \frac{1}{1-\kappa_{1}}-\frac{\partial w_{1}^{\gamma}}{\partial x}\right) \text { on } \dot{\mathcal{S}}^{1}, \\
& \gamma \delta \leq \min \left(w_{1}^{\gamma}, \frac{1}{1-\kappa_{1}}-\frac{\partial w_{1}^{\gamma}}{\partial x}\right) \text { on }\{0\} \times[0, \delta] \\
& \gamma \delta \leq \min \left(w_{0}^{\gamma}(0), \frac{1}{1-\kappa_{0}}-\left(w_{0}^{\gamma}\right)^{\prime}(0)\right)
\end{aligned}
$$

and $u_{i}^{\gamma}:=-\gamma h+(1+\gamma) u_{i},(i=0,1)$ is a strict viscosity subsolution of 3.17-3.20:

$$
\begin{aligned}
&-\gamma \delta \geq \min \left(\rho u_{0}^{\gamma}-\mathcal{L} u_{0}^{\gamma}-\mathcal{J}\left(u_{0}^{\gamma}, u_{1}^{\gamma}\right), \mathbb{1}_{[0, D)}+\mathbb{1}_{[D,+\infty)}\left[\left(u_{0}^{\gamma}\right)^{\prime}-\frac{1}{1+\kappa^{\prime}}\right]\right.\left.\frac{1}{1-\kappa_{0}}-\left(u_{0}^{\gamma}\right)^{\prime}\right) \text { on } \mathcal{S}^{0}, \\
&-\gamma \delta \geq \min \left(\rho u_{1}^{\gamma}-\mathcal{L} u_{1}^{\gamma}-\frac{\partial u_{1}^{\gamma}}{\partial \varsigma}, \frac{1}{1-\kappa_{1}}-\frac{\partial u_{1}^{\gamma}}{\partial x}\right) \text { on } \dot{\mathcal{S}}^{1}, \\
&-\gamma \delta \geq \min \left(u_{1}^{\gamma}, \frac{1}{1-\kappa_{1}}-\frac{\partial u_{1}^{\gamma}}{\partial x}\right) \text { on }\{0\} \times[0, \delta], \\
&-\gamma \delta \geq \min \left(u_{0}^{\gamma}(0), \frac{1}{1-\kappa_{0}}-\left(u_{0}^{\gamma}\right)^{\prime}(0)\right) .
\end{aligned}
$$

Assume that

$$
M:=\sup _{(t, x) \in \mathcal{S}, i=0,1} u_{i}^{\gamma}(t, x)-w_{i}^{\gamma}(t, x)>0 .
$$


First, we note that

$$
\lim _{x \rightarrow \infty} u_{0}^{\gamma}(x)-w_{0}^{\gamma}(x)=-\infty .
$$

This is due to the fact that $w_{0}$ is a supersolution and $u_{0}$ is a subsolution so that there exists $C>0$ such that $w_{0}(x) \geq \frac{x}{1+\kappa^{\prime}}$ and $u_{0}(x) \leq C+\frac{x}{1+\kappa^{\prime}}$ for $x$ large enough. Hence we know that $M=u_{i}^{\gamma}\left(t_{0}, x_{0}\right)-w_{i}^{\gamma}\left(t_{0}, x_{0}\right)$ for some $\left(t_{0}, x_{0}\right) \in \mathcal{S}$ and some $i=0,1$.

(a) Suppose $i=0$. For $\epsilon>0$, define $\Phi^{\epsilon}(x, y)=u_{0}^{\gamma}(x)-w_{0}^{\gamma}(y)-\phi_{\epsilon}(x, y)$ with

$$
\phi_{\epsilon}(x, y)=\frac{1}{4}\left|x-x_{0}\right|^{4}+\frac{1}{2 \epsilon}|x-y|^{2}+\frac{1}{1-\kappa_{0}}(x-y) .
$$

For each $\epsilon>0$, the function $\Phi^{\epsilon}$ attains its maximum value $M_{\epsilon}$ at some point $\left(x_{\epsilon}, y_{\epsilon}\right) \epsilon$ $\mathcal{S}^{0} \times \mathcal{S}^{0}$. Classical results in comparison theory for viscosity solutions show that by taking a subsequence, we can assume that the sequence $\left(x_{\epsilon}, y_{\epsilon}\right)$ converges to $\left(x_{0}, x_{0}\right)$ as $\epsilon \rightarrow 0$, and

$$
\lim _{\epsilon \rightarrow 0} \frac{\left|x_{\epsilon}-y_{\epsilon}\right|}{\epsilon}=0
$$

Consequently, $M_{\epsilon} \rightarrow M$ as $\epsilon \rightarrow 0$, and $M_{\epsilon}>0$ for $\epsilon$ small enough. By Theorem 3.2 of [8], there exist $\xi_{\epsilon}, \zeta_{\epsilon} \in \mathbb{R}$ such that

$$
\begin{gathered}
\left(\left(x_{\epsilon}-y_{\epsilon}\right) / \epsilon+\left(x_{\epsilon}-x_{0}\right)^{3}+\frac{1}{1-\kappa_{0}}, \xi_{\epsilon}\right) \in J^{2,+} u_{0}\left(x_{\epsilon}\right), \\
\left(\left(x_{\epsilon}-y_{\epsilon}\right) / \epsilon+\frac{1}{1-\kappa_{0}}, \zeta_{\epsilon}\right) \in J^{2,-} w_{0}^{\gamma}\left(y_{\epsilon}\right),
\end{gathered}
$$

and

$$
\left(\begin{array}{cc}
\xi_{\epsilon} & 0 \\
0 & \zeta_{\epsilon}
\end{array}\right) \leq D^{2} \phi_{\epsilon}\left(x_{\epsilon}, y_{\epsilon}\right)+\epsilon\left(D^{2} \phi_{\epsilon}\left(x_{\epsilon}, y_{\epsilon}\right)\right)^{2}
$$

which implies that

$$
\begin{aligned}
\sigma\left(x_{\epsilon}\right)^{2} \xi_{\epsilon}-\sigma\left(y_{\epsilon}\right)^{2} \zeta_{\epsilon} \leq & \frac{3}{\epsilon}\left(\sigma\left(x_{\epsilon}\right)-\sigma\left(y_{\epsilon}\right)\right)^{2}-6 \sigma\left(x_{\epsilon}\right) \sigma\left(y_{\epsilon}\right)\left(x_{\epsilon}-x_{0}\right)^{2} \\
& +9 \sigma\left(x_{\epsilon}\right)^{2}\left(x_{\epsilon}-x_{0}\right)^{2}+9 \epsilon \sigma\left(x_{\epsilon}\right)^{2}\left(x_{\epsilon}-x_{0}\right)^{4} \\
& \leq \frac{C}{\epsilon}\left|x_{\epsilon}-y_{\epsilon}\right|^{2}+C\left(1+x_{0}^{2}\right)\left(\left|x_{\epsilon}-x_{0}\right|^{2}+\left|x_{\epsilon}-x_{0}\right|^{4}\right) .
\end{aligned}
$$

If $y_{\epsilon}=0$, then by $(5.59)$

$$
\gamma \delta \leq \min \left(w_{0}^{\gamma}\left(y_{\epsilon}\right),-\left(x_{\epsilon}-y_{\epsilon}\right) / \epsilon\right) .
$$

By sending $\epsilon$ to 0 , we obtain a contradiction for $\epsilon$ small because of (5.65). If $x_{\epsilon}=0$, we find

$$
-\gamma \delta \geq \min \left(u_{0}^{\gamma}(0),-\left(x_{\epsilon}-y_{\epsilon}\right) / \epsilon-\left(x_{\epsilon}-x_{0}\right)^{3}\right)
$$


Therefore, when $\epsilon$ is small enough, $u_{0}^{\gamma}\left(x_{\epsilon}\right) \leq-\gamma \delta$. A contradiction arises from the fact that $w_{0}^{\gamma}\left(y_{\epsilon}\right) \geq 0$ but $M_{\epsilon}=u_{0}^{\gamma}\left(x_{\epsilon}\right)-w_{0}^{\gamma}\left(y_{\epsilon}\right)>0$. Consequently, $x_{0}>0$ and $x_{\epsilon}, y_{\epsilon}>0$ for $\epsilon$ large enough.

By (5.60) and (5.66), we get

$$
\begin{aligned}
& \min \left(\rho u_{0}^{\gamma}\left(x_{\epsilon}\right)-\mu\left(x_{\epsilon}\right) p_{\epsilon}-\frac{1}{2} \sigma\left(x_{\epsilon}\right)^{2} \xi_{\epsilon}-\mathcal{J}\left(u_{0}^{\gamma}\left(x_{\epsilon}\right), u_{1}^{\gamma}\left(x_{\epsilon}\right)\right),\right. \\
& \left.\mathbb{1}_{[0, D)}+\mathbb{1}_{[D,+\infty)}\left(p_{\epsilon}-\frac{1}{1+\kappa^{\prime}}\right),-\left(x_{\epsilon}-y_{\epsilon}\right) / \epsilon-\left(x_{\epsilon}-x_{0}\right)^{3}\right) \leq-\gamma \delta,
\end{aligned}
$$

with $p_{\epsilon}=\left(x_{\epsilon}-y_{\epsilon}\right) / \epsilon+\left(x_{\epsilon}-x_{0}\right)^{3}+\frac{1}{1-\kappa_{0}}$. By sending $\epsilon$ to 0 , we see that the only possibility is

$$
\rho u_{0}^{\gamma}\left(x_{\epsilon}\right)-\mu\left(x_{\epsilon}\right) p_{\epsilon}-\frac{1}{2} \sigma\left(x_{\epsilon}\right)^{2} \xi_{\epsilon}-\mathcal{J}\left(u_{0}^{\gamma}\left(x_{\epsilon}\right), u_{1}^{\gamma}\left(x_{\epsilon}\right)\right) \leq-\gamma \delta
$$

By (5.56) and (5.67), we also have

$$
\rho w_{0}^{\gamma}\left(y_{\epsilon}\right)-\mu\left(y_{\epsilon}\right)\left(\left(x_{\epsilon}-y_{\epsilon}\right) / \epsilon+\frac{1}{1-\kappa_{0}}\right)-\frac{1}{2} \sigma\left(y_{\epsilon}\right)^{2} \zeta_{\epsilon}-\mathcal{J}\left(w_{0}^{\gamma}\left(y_{\epsilon}\right), w_{1}^{\gamma}\left(y_{\epsilon}\right)\right) \geq \gamma \delta .
$$

Combining these last two inequalities, we get

$$
\begin{aligned}
\rho\left(w_{0}^{\gamma}\left(y_{\epsilon}\right)-u_{0}^{\gamma}\left(x_{\epsilon}\right)\right) \geq & 2 \gamma \delta+\left(\mu\left(y_{\epsilon}\right)-\mu\left(x_{\epsilon}\right)\right)\left(\left(x_{\epsilon}-y_{\epsilon}\right) / \epsilon+\frac{1}{1-\kappa_{0}}\right)-\mu\left(x_{\epsilon}\right)\left(x_{\epsilon}-x_{0}\right)^{3} \\
& +\frac{1}{2} \sigma\left(y_{\epsilon}\right)^{2} \zeta_{\epsilon}-\frac{1}{2} \sigma\left(x_{\epsilon}\right)^{2} \xi_{\epsilon}+\mathcal{J}\left(w_{0}^{\gamma}\left(y_{\epsilon}\right), w_{1}^{\gamma}\left(y_{\epsilon}\right)\right)-\mathcal{J}\left(u_{0}^{\gamma}\left(x_{\epsilon}\right), u_{1}^{\gamma}\left(x_{\epsilon}\right)\right) .
\end{aligned}
$$

The right side of this inequality converges to

$$
2 \gamma \delta+\lambda \mathbb{1}_{[0, D]}\left(w_{1}^{\gamma}\left(x_{0}, 0\right)-w_{0}^{\gamma}\left(x_{0}\right)-u_{1}^{\gamma}\left(x_{0}, 0\right)+u_{0}^{\gamma}\left(x_{0}\right)\right)
$$

which is bigger or equal to $2 \gamma \delta$ since

$$
u_{0}^{\gamma}\left(x_{0}\right)-w_{0}^{\gamma}\left(x_{0}\right)=M \geq u_{1}^{\gamma}\left(x_{0}, 0\right)-w_{1}^{\gamma}\left(x_{0}, 0\right)
$$

On the other hand, the left side converges to $-\rho M<0$, hence the contradiction.

(b) Suppose $i=1$.

If $t_{0}=\delta$ or $x_{0}=D$, then

$$
\begin{aligned}
u_{1}^{\gamma}\left(t_{0}, x_{0}\right)-w_{1}^{\gamma}\left(t_{0}, x_{0}\right)= & (1-\gamma) \max \left(u_{0}(D)-\left(D-x_{0}\right) /\left(1-\kappa_{1}\right), 0\right) \\
& \quad-(1-\gamma) \max \left(w_{0}(D)-\left(D-x_{0}\right) /\left(1-\kappa_{1}\right), 0\right)-2 \gamma h\left(x_{0}\right) \\
\leq & (1-\gamma) \max \left(u_{0}(D)-w_{0}(D), 0\right)-2 \gamma h\left(x_{0}\right) \\
\leq & \max \left(u_{0}^{\gamma}(D)-w_{0}^{\gamma}(D), 0\right) .
\end{aligned}
$$


This is therefore equivalent to the case $i=0, x_{0}=D$, which we have already treated.

We can therefore assume $x_{0}<D$ and $t_{0}<\delta$.

For $\epsilon>0$, define $\Phi^{\epsilon}(t, x, y)=u_{1}^{\gamma}(t, x)-w_{1}^{\gamma}(t, y)-\phi_{\epsilon}(t, x, y)$ with

$$
\phi_{\epsilon}(t, x, y)=\frac{1}{2}\left|t-t_{0}\right|^{2}+\frac{1}{4}\left|x-x_{0}\right|^{4}+\frac{1}{2 \epsilon}|x-y|^{2}+\frac{1}{1-\kappa_{1}}(x-y) .
$$

For each $\epsilon>0$, the function $\Phi^{\epsilon}$ attains a maximum at $\left(t_{\varepsilon}, x_{\epsilon}, y_{\epsilon}\right)$. By taking a subsequence, we can assume that the sequence $\left(t_{\varepsilon}, x_{\epsilon}, y_{\epsilon}\right)$ converges to $\left(t_{0}, x_{0}, x_{0}\right)$ as $\epsilon \rightarrow 0$, and

$$
\lim _{\epsilon \rightarrow 0} \frac{\left|x_{\epsilon}-y_{\epsilon}\right|}{\epsilon}=0
$$

For $\epsilon$ small enough, we can therefore assume $x_{\epsilon}, y_{\epsilon}<D$ and $t_{\epsilon}<\delta$. By Theorem 8.3 of [8], there exist $b_{\epsilon}, \xi_{\epsilon}, \zeta_{\epsilon} \in \mathbb{R}$ such that

$$
\begin{gathered}
\left(b_{\epsilon}+2\left(t-t_{0}\right),\left(x_{\epsilon}-y_{\epsilon}\right) / \epsilon+\left(x_{\epsilon}-x_{0}\right)^{3}+\frac{1}{1-\kappa_{1}}, \xi_{\epsilon}\right) \in J^{2,+} u_{0}\left(t_{\epsilon}, x_{\epsilon}\right), \\
\left(b_{\epsilon},\left(x_{\epsilon}-y_{\epsilon}\right) / \epsilon+\frac{1}{1-\kappa_{1}}, \zeta_{\epsilon}\right) \in J^{2,-} w_{0}^{\gamma}\left(t_{\epsilon}, y_{\epsilon}\right)
\end{gathered}
$$

and

$$
\sigma\left(x_{\epsilon}\right)^{2} \xi_{\epsilon}-\sigma\left(y_{\epsilon}\right)^{2} \zeta_{\epsilon} \leq \frac{C}{\epsilon}\left|x_{\epsilon}-y_{\epsilon}\right|^{2}+C\left(1+x_{0}^{2}\right)\left(\left|x_{\epsilon}-x_{0}\right|^{2}+\left|x_{\epsilon}-x_{0}\right|^{4}\right)
$$

as before. Furthermore, we can show that $x_{\epsilon}, y_{\epsilon}>0$ for $\epsilon$ small enough. Therefore,

$$
\min \left(\rho u_{1}^{\gamma}\left(x_{\epsilon}\right)-\mu\left(x_{\epsilon}\right) p_{\epsilon}^{\prime}-\frac{1}{2} \sigma\left(x_{\epsilon}\right)^{2} \xi_{\epsilon}-b_{\epsilon}-2\left(t-t_{0}\right),-\left(x_{\epsilon}-y_{\epsilon}\right) / \epsilon-\left(x_{\epsilon}-x_{0}\right)^{3}\right) \leq-\gamma \delta
$$

with $p_{\epsilon}^{\prime}=\left(x_{\epsilon}-y_{\epsilon}\right) / \epsilon+\left(x_{\epsilon}-x_{0}\right)^{3}+\frac{1}{1-\kappa_{1}}$, and

$$
\left.\rho w_{0}^{\gamma}\left(y_{\epsilon}\right)-\mu\left(y_{\epsilon}\right)\left(\left(x_{\epsilon}-y_{\epsilon}\right) / \epsilon+\frac{1}{1-\kappa_{1}}\right)-\frac{1}{2} \sigma\left(y_{\epsilon}\right)^{2} \zeta_{\epsilon}-b_{\epsilon},-\left(x_{\epsilon}-y_{\epsilon}\right) / \epsilon\right) \geq \gamma \delta .
$$

As before, we find a contradiction by sending $\epsilon$ to 0 . 


\section{References}

[1] S. Asmussen and M. Taksar (1997), Controlled diffusion models for optimal dividend pay-out, Insurance Math. Econom., 20, pp. 1-15.

[2] F. Black and J. C. Cox (1976), Valuing Corporate Securities: Some Effects of Bond Indenture Provisions, Journal of Finance, 31, 351-367.

[3] M. Broadie, M. Chernov, and S. Sundaresan (2007), Optimal debt and equity values in the presence of Chapter 7 and Chapter 11, Journal of Finance, 62(3), pp. 1341-1377.

[4] E. Chevalier, V. Ly Vath and S. Scotti (2013), An optimal dividend and investment control problem under debt constraints, SIAM J. Financial Math., 4, pp. 297-326.

[5] E. Chevalier, M. Gaigi, and V. Ly Vath (2017), Liquidity risk and optimal dividend/investment strategies, Math. Financ. Econ., 11 (1), pp. 111-135.

[6] T. Choulli, M. Taksar, and X.Y. Zhou (2003), A diffusion model for optimal dividend distribution for a company with constraints on risk control, SIAM J. Control Optim., 41, 1946-1979.

[7] T. Choulli, M. Taksar, and X.Y. Zhou (2004), Interplay between dividend rate and business constraints for a financial corporation, Ann. Appl. Probab,14, pp. 1810-1837.

[8] M. Crandall, H. Ishii and P.L. Lions (1992), User's guide to viscosity solutions of second order partial differential equations, Bull. Amer. Math. Soc., 27, pp. 1-67.

[9] J.P. Décamps and S. Villeneuve (2007), Optimal dividend policy and growth option, Finance and Stoch., 11(1), pp. 3-27.

[10] X. Guo and H. Pham (2005): "Optimal partially reversible investment with entry decision and general production function", Stochastic Processes and their Applications 115, 705-736.

[11] M. Jeanblanc and A. Shiryaev (1995), Optimization of the flow of dividends, Russian Math. Surveys, 50, pp. 257-277.

[12] Jin Z., G. Yin, Zhu C. : Numerical solutions of optimal risk control and dividend optimization policies under a generalized singular control formulation. Automatica, 48(8), 1489-1501 (2012).

[13] Z. Jin and G. Yin (2013), An optimal dividend policy with delayed capital injections, ANZIAM J., 55, pp. 129-150.

[14] Z. Jin, H. Yang, G. Yin (2013): Numerical methods for optimal dividend payment and investment strategies of regime-switching jump diffusion models with capital injections, Automatica, 49, pp. 2317-2329. 
[15] J. Keppo and S. Peura (2006), Optimal Bank Capital with Costly Recapitalization, The Journal of Business, Vol. 79, No. 4, pp. 2163-2201

[16] A. Lokka and M. Zervos (2008), Optimal dividend and issuance of equity policies in the presence of proportional costs, Insurance Math. Econom., 42, pp. 954-961.

[17] V. Ly Vath, H. Pham and S. Villeneuve (2008), A Mixed Singular/Switching Control Problem For A Dividend Policy With Reversible Technology Investment, Ann. Appl. Probab., 18, pp. 1164-1200.

[18] R. N. Makarov, A. Metzler and Z. Ni (2015), Modelling default risk with occupation times, Finance Research Letters, 13, pp. 54-65.

[19] R. C. Merton (1974), On the pricing of corporate debt: the risk structure of interest rates, Journal of Finance, 29, pp. 449-70.

[20] G. Peskir (2006), On reflecting Brownian motion with drift, Proc. Symp.Stoch.Syst., ISCIE Kyoto, pp. 1-5.

[21] S. P. Sethi and M. I. Taksar (2002), Optimal financing of a corporation subject to random returns, Math. Finance, 12, pp. 155-172.

[22] Y. Yildirim (2006), Modeling default risk: a new structural approach, Finance Research Letters, 3, pp. 165-172 